\title{
Modeling of GERDA Phase II data
}

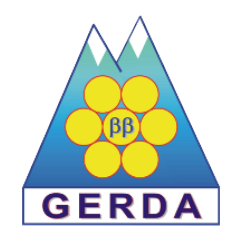

\section{The GERDA collaboration}

M. Agostini, ${ }^{p}$ A.M. Bakalyarov, ${ }^{n}$ M. Balata, ${ }^{a}$ I. Barabanov, ${ }^{l}$ L. Baudis, ${ }^{t}$ C. Bauer, ${ }^{h}$ E. Bellotti, ${ }^{i, j}$ S. Belogurov, ${ }^{m, l, 1}$ A. Bettini, ${ }^{q, r}$ L. Bezrukov, ${ }^{l}$ D. Borowicz, ${ }^{f}$ E. Bossio, ${ }^{p}$ V. Bothe, ${ }^{h}$ V. Brudanin, ${ }^{f}$ R. Brugnera, ${ }^{q, r}$ A. Caldwell, ${ }^{o}$ C. Cattadori, ${ }^{j}$

A. Chernogorov, ${ }^{m, n}$ T. Comellato, ${ }^{p}$ V. D'Andrea, ${ }^{b}$ E.V. Demidova, ${ }^{m}$ N. Di Marco, ${ }^{a}$

A. Domula, ${ }^{e}$ E. Doroshkevich, ${ }^{l}$ V. Egorov, ${ }^{f, 2}$ F. Fischer, ${ }^{o}$ M. Fomina, ${ }^{f}$

A. Gangapshev, ${ }^{l, h}$ A. Garfagnini, ${ }^{q, r}$ C. Gooch, ${ }^{o}$ P. Grabmayr, ${ }^{s}$ V. Gurentsov, ${ }^{l}$

K. Gusev, ${ }^{f, n, p}$ J. Hakenmüller, ${ }^{h}$ S. Hemmer, ${ }^{r}$ R. Hiller, ${ }^{t}$ W. Hofmann, ${ }^{h}$ M. Hult, ${ }^{g}$

L.V. Inzhechik, ${ }^{l, 3}$ J. Janicskó Csáthy, ${ }^{p, 4}$ J. Jochum, ${ }^{s}$ M. Junker, ${ }^{a}$ V. Kazalov, ${ }^{l}$

Y. Kermaïdic, ${ }^{h}$ T. Kihm, ${ }^{h}$ I.V. Kirpichnikov, ${ }^{m}$ A. Klimenko, ${ }^{h, f, 5}$ R. KneißI, ${ }^{o}$

K.T. Knöpfle, ${ }^{h}$ O. Kochetov, ${ }^{f}$ V.N. Kornoukhov, ${ }^{m, l}$ P. Krause, ${ }^{p}$ V.V. Kuzminov, ${ }^{l}$

M. Laubenstein, ${ }^{a}$ A. Lazzaro, ${ }^{p}$ M. Lindner, ${ }^{h}$ I. Lippi, ${ }^{r}$ A. Lubashevskiy, ${ }^{f}$

B. Lubsandorzhiev, ${ }^{l}$ G. Lutter, ${ }^{g}$ C. Macolino, ${ }^{a, 6}$ B. Majorovits, ${ }^{o}$ W. Maneschg, ${ }^{h}$

M. Miloradovic, ${ }^{t}$ R. Mingazheva, ${ }^{t}$ M. Misiaszek, ${ }^{d}$ P. Moseev, ${ }^{l}$ I. Nemchenok, ${ }^{f, 5}$

K. Panas, ${ }^{d}$ L. Pandola, ${ }^{c}$ K. Pelczar, ${ }^{a}$ L. Pertoldi, ${ }^{q, r}$ P. Piseri, ${ }^{k}$ A. Pullia, ${ }^{k}$ C. Ransom, ${ }^{t}$

S. Riboldi, ${ }^{k}$ N. Rumyantseva, ${ }^{n, f}$ C. Sada, ${ }^{q, r}$ F. Salamida, ${ }^{b}$ S. Schönert, ${ }^{p}$ J. Schreiner, ${ }^{h}$

M. Schütt, ${ }^{h}$ A-K. Schütz, ${ }^{s}$ O. Schulz, ${ }^{o}$ M. Schwarz, ${ }^{p}$ B. Schwingenheuer, ${ }^{h}$

O. Selivanenko, ${ }^{l}$ E. Shevchik, ${ }^{f}$ M. Shirchenko, ${ }^{f}$ H. Simgen, ${ }^{h}$ A. Smolnikov, ${ }^{h, f}$

D. Stukov, ${ }^{n}$ L. Vanhoefer, ${ }^{o}$ A.A. Vasenko, ${ }^{m}$ A. Veresnikova, ${ }^{l}$ C. Vignoli, ${ }^{a}$

K. von Sturm, ${ }^{q, r}$ T. Wester, ${ }^{e}$ C. Wiesinger, ${ }^{p}$ M. Wojcik, ${ }^{d}$ E. Yanovich, ${ }^{l}$ B. Zatschler, ${ }^{e}$

I. Zhitnikov, ${ }^{f}$ S.V. Zhukov, ${ }^{n}$ D. Zinatulina, ${ }^{f}$ A. Zschocke, ${ }^{s}$ A.J. Zsigmond, ${ }^{o}$

K. Zuber ${ }^{e}$ and G. Zuzel ${ }^{d}$

\footnotetext{
${ }^{1}$ Also at: NRNU MEPhI, Moscow, Russia.

${ }^{2}$ Deceased.

${ }^{3}$ Also at: Moscow Inst. of Physics and Technology, Russia.

${ }^{4}$ Present address: Leibniz-Institut für Kristallzüchtung, Berlin, Germany.

${ }^{5}$ Also at: Dubna State University, Dubna, Russia.

${ }^{6}$ Present address: LAL, CNRS/IN2P3, Université Paris-Saclay, Orsay, France.
} 
${ }^{a}$ INFN Laboratori Nazionali del Gran Sasso and Gran Sasso Science Institute, Assergi, Italy

${ }^{b}$ INFN Laboratori Nazionali del Gran Sasso and Università degli Studi dell'Aquila, L'Aquila, Italy

${ }^{c}$ INFN Laboratori Nazionali del Sud, Catania, Italy

${ }^{d}$ Institute of Physics, Jagiellonian University, Cracow, Poland

${ }^{e}$ Institut für Kern- und Teilchenphysik, Technische Universität Dresden, Dresden, Germany

${ }^{f}$ Joint Institute for Nuclear Research, Dubna, Russia

${ }^{g}$ European Commission, JRC-Geel, Geel, Belgium

${ }^{h}$ Max-Planck-Institut für Kernphysik, Heidelberg, Germany

${ }^{i}$ Dipartimento di Fisica, Università Milano Bicocca, Milan, Italy

${ }^{j}$ INFN Milano Bicocca, Milan, Italy

${ }^{k}$ Dipartimento di Fisica, Università degli Studi di Milano and INFN Milano, Milan, Italy

${ }^{l}$ Institute for Nuclear Research of the Russian Academy of Sciences, Moscow, Russia

${ }^{m}$ Institute for Theoretical and Experimental Physics, NRC "Kurchatov Institute", Moscow, Russia

${ }^{n}$ National Research Centre "Kurchatov Institute", Moscow, Russia

${ }^{\circ}$ Max-Planck-Institut für Physik, Munich, Germany

${ }^{p}$ Physik Department, Technische Universität München, Germany

${ }^{q}$ Dipartimento di Fisica e Astronomia, Università degli Studi di Padova, Padua, Italy

${ }^{r}$ INFN Padova, Padua, Italy

${ }^{s}$ Physikalisches Institut, Eberhard Karls Universität Tübingen, Tübingen, Germany

${ }^{t}$ Physik-Institut, Universität Zürich, Zurich, Switzerland

E-mail: gerda-eb@mpi-hd.mpg.de

Abstract: The GERmanium Detector Array (Gerda) experiment at the Gran Sasso underground laboratory (LNGS) of INFN is searching for neutrinoless double-beta $(0 \nu \beta \beta)$ decay of ${ }^{76} \mathrm{Ge}$. The technological challenge of GERDA is to operate in a "backgroundfree" regime in the region of interest (ROI) after analysis cuts for the full $100 \mathrm{~kg} \cdot \mathrm{yr}$ target exposure of the experiment. A careful modeling and decomposition of the full-range energy spectrum is essential to predict the shape and composition of events in the ROI around $Q_{\beta \beta}$ for the $0 \nu \beta \beta$ search, to extract a precise measurement of the half-life of the doublebeta decay mode with neutrinos $(2 \nu \beta \beta)$ and in order to identify the location of residual impurities. The latter will permit future experiments to build strategies in order to further lower the background and achieve even better sensitivities. In this article the background decomposition prior to analysis cuts is presented for GERDA Phase II. The background model fit yields a flat spectrum in the ROI with a background index (BI) of $16.04_{-0.85}^{+0.78}$. $10^{-3} \mathrm{cts} /(\mathrm{keV} \cdot \mathrm{kg} \cdot \mathrm{yr})$ for the enriched BEGe data set and $14.68_{-0.52}^{+0.47} \cdot 10^{-3} \mathrm{cts} /(\mathrm{keV} \cdot \mathrm{kg} \cdot \mathrm{yr})$ for the enriched coaxial data set. These values are similar to the one of Phase I despite a much larger number of detectors and hence radioactive hardware components.

Keywords: Dark Matter and Double Beta Decay (experiments)

ARXIV EPRINT: 1909.02522 


\section{Contents}

1 Introduction 1

2 Data selection and prior knowledge 2

2.1 Analysis data sets 4

2.2 Monte Carlo simulations and probability density functions 4

2.3 Background expectation 5

$\begin{array}{lll}3 & \text { Statistical analysis } & 10\end{array}$

$\begin{array}{ll}3.1 \text { Analysis window and binning } & 10\end{array}$

$\begin{array}{ll}3.2 \text { Likelihood factorization } & 11\end{array}$

$\begin{array}{lll}3.3 & \text { Prior distributions } & 12\end{array}$

4 Results 12

$\begin{array}{lll}5 & \text { Discussion } & 17\end{array}$

$\begin{array}{llr}6 & \text { Conclusions } & 19\end{array}$

$\begin{array}{lr}\text { A Potassium tracking analysis } & \mathbf{2 0}\end{array}$

$\begin{array}{lll}\text { A.1 Data } & 21\end{array}$

$\begin{array}{lll}\text { A.2 Analysis } & 22\end{array}$

$\begin{array}{ll}\text { B } \alpha \text { events background analysis } & 27\end{array}$

B.1 Time distribution of $\alpha$ events 30

C Monte Carlo simulations and probability density functions 32

\section{Introduction}

A large fraction of current experimental efforts are devoted to test the precision of the standard model of particle physics and investigate the presence of new phenomena. Many extensions of the standard model predict rare processes and in particular the existence of neutrinoless double-beta $(0 \nu \beta \beta)$ decay [1-3]. The observation of this lepton-number violating decay would shed light on the nature of neutrinos and could give a hint on the scale of neutrino masses.

The GERmanium Detector Array (GERDA) experiment [4, 5] is searching for the $0 \nu \beta \beta$ decay of the candidate isotope ${ }^{76} \mathrm{Ge}$ at a $\mathrm{Q}$-value of $Q_{\beta \beta}=2039.061(7) \mathrm{keV}$ [6]. GerdA is operating 37 detectors made from material enriched in ${ }^{76} \mathrm{Ge}$ and a total mass of $35.6 \mathrm{~kg}$ bare in $64 \mathrm{~m}^{3}$ of liquid Argon (LAr, purity 5.0). The experiment profits from the high shielding 
power of the LAr and its scintillation properties. A hybrid instrumentation consisting of light guiding fibers read out by silicon photomultipliers (SiPMs) and 16 photomultipliers (PMTs) detect LAr scintillation light in order to veto events depositing energy in the cryogenic liquid [5]. The LAr cryostat itself is situated inside a tank filled with $590 \mathrm{~m}^{3}$ of purified water shielding against external ionizing radiation and neutrons. Furthermore, it is instrumented with 66 PMTs to veto muons by the detection of Cerenkov light. GERDA is the first $0 \nu \beta \beta$ decay experiment working in a "background-free" regime in the region of interest (ROI) after analysis cuts [7-9], where the ROI is $Q_{\beta \beta} \pm \mathrm{FWHM} / 2$, and FWHM is defined as full width half maximum.

In the following, we present the spectral decomposition of GERDA Phase II data. The analysis is conducted prior the application of active background suppression techniques to data, i.e. the LAr veto [5] and pulse shape discrimination (PSD) taking advantage of particular detector signal shapes [10]. A new assay of the GERDA background is necessary due to substantial upgrade works finished in 2015 [5]. Most structural components close to the detectors have been exchanged using materials with improved radio-purity, the detector array has been enlarged and the LAr veto instrumentation has been deployed during the upgrade. Moreover, each detector string (enclosed in a copper mini-shroud during Phase I) has been encapsulated in a transparent nylon mini-shroud in order to limit the drift of ${ }^{42} \mathrm{~K}$ ions in the detector vicinity and appropriately propagate the LAr scintillation light [11] (see section 2.3 for details). The introduction of these new setup components and materials changes the distribution and composition of radioactive impurities in the setup.

A precise knowledge of the spectral composition of the data is a key point for further analysis like accessing the half-life of the lepton number conserving mode of double-beta $(2 \nu \beta \beta)$ decay. Moreover, there are significant efforts towards reaching the tonne-scale of active isotope mass and the localization of remaining radioactive impurities inside the setup is the basis for the possible further reduction of background. This is essential for future endeavors in order to boost the current signal discovery and limit setting sensitivity by two orders of magnitude to the range of $T_{1 / 2}^{0 \nu}>1 \cdot 10^{28} \mathrm{yr}$.

\section{Data selection and prior knowledge}

The data analyzed in the following were taken between December 2015 and April 2018. In this period the GERDA array consisted of 40 high-purity germanium (HPGe) detectors: 30 Broad Energy Germanium (BEGe) detectors [12,13] and 10 detectors with a semi-coaxial geometry three of which are made from germanium with a natural isotope composition. The enrichment fraction of the 30 enriched BEGe ( ${ }^{\mathrm{enr}} \mathrm{BEGe}$ ) detectors is $87.8 \%$ while the respective fraction for the 7 enriched coaxial ( ${ }^{\mathrm{enr}} \mathrm{Coax}$ ) detectors is in the range of $85.5-88.3 \%[5]$.

Detector geometries. The GERDA HPGe detectors are made of p-type germanium. $\mathrm{p}^{+}$ and $\mathrm{n}^{+}$contacts are manufactured via boron implantation and lithium diffusion, respectively. The $\mathrm{p}^{+}$electrode is connected to a charge sensitive amplifier while the $\mathrm{n}^{+}$electrode is biased at typically $4 \mathrm{kV}$. A groove between the two contacts provides electrical insulation. The bias high-voltage creates an internal electrical field which is responsible for charge 
collection. When biased at full-depletion voltage, the germanium detectors reach maximal $(\varepsilon=1)$ charge collection efficiency $(\mathrm{CCE})$ in an internal active volume, surrounded by a transition layer (TL) with reduced CCE $(0<\varepsilon<1)$ and low electric field. The TL is covered by a thin conductive layer in which all charges recombine and charge collection is entirely suppressed $(\varepsilon=0)$, therefore, called dead layer. We define the contact thickness as the depth at which the CCE reaches its maximal value. The GERDA detectors are of two distinct geometries. In the semi-coaxial layout the thin $\mathrm{p}^{+}$contact $(0.5-1 \mu \mathrm{m})$ covers the entire bore hole; in the BEGe-type, instead, the same contact is a disk of $15 \mathrm{~mm}$ diameter (see figure 3 in reference [14]). The $\mathrm{n}^{+}$contact, about $1 \mathrm{~mm}$ thick, "wraps around" the detector. An exhaustive description of the GERDA detector geometries and properties can be found in previous publications $[5,12-14]$. The detector arrangement in the 7 strings that constitute the GERDA array is graphically presented in figure 1a (and in the appendix in figure 8).

Data acquisition and treatment. All data are recorded using FADCs and are digitally processed off-line [5]. The linearity of the data acquisition system and off-line energy reconstruction was tested with a precision pulse generator over the whole dynamic range of the FADCs. Up to an energy of at least $6 \mathrm{MeV}$ no major non-linearity and pulse shape deformation was observed.

A signal above threshold in any of the germanium detectors triggers the data acquisition and the respective event is written to disk. An event is defined as the set of traces recorded in the 40 germanium detectors, 16 photomultipliers (PMT) and 15 silicon photomultiplier (SiPM) channels from the LAr veto and the signal from the Water Cerenkov muon veto. This hardware threshold is detector and run dependent and varies between $20 \mathrm{keV}$ and $200 \mathrm{keV}$. The energies of all other detectors are reconstructed from the recorded traces and we apply a threshold of $40 \mathrm{keV}$ on these. At this threshold the reconstruction efficiency is practically $100 \%$. Hence, we are sure to treat data and simulations in a consistent manner. In the following, we define the multiplicity of an event as the number of germanium detectors in which an energy of at least $40 \mathrm{keV}$ is registered.

The energy deposition associated to each germanium detector signal is determined via a zero area cusp (ZAC) filter [16] which is optimized off-line for each detector and each calibration. Calibrations are usually taken with three ${ }^{228} \mathrm{Th}$ sources which are lowered into the LAr to the vicinity of the detector array in a 1-2 week cycle. An energy correction due to crosstalk between detector channels is performed for each event. The average crosstalk for all pairs of channels is about $0.05 \%$. Details about the crosstalk correction can be found in reference [17]. Events in a window $Q_{\beta \beta} \pm 25 \mathrm{keV}$ are excluded from the analysis until all selection cuts are finalized. The number of events and their energies in this window are only released once all analysis steps are defined.

Each event has to pass a number of quality cuts which are tailored to filter unphysical events [7]. Data taking periods in which stable operation cannot be guaranteed are excluded from analysis. The overall duty cycle in GERDA Phase II is $92.9 \%$. We keep $80.4 \%$ of the recorded data as valid analysis data set, discarding for instance, periods of unstable calibration or earthquakes like in August 2016. Detectors with an unstable energy calibra- 


\begin{tabular}{|lccccc|}
\hline data set & composition & $\begin{array}{c}\text { total Ge } \\
\text { mass }[\mathrm{kg}]\end{array}$ & $\begin{array}{c}\text { active }{ }^{76} \mathrm{Ge} \\
\text { mass }[\mathrm{kg}]\end{array}$ & $\begin{array}{c}\text { total Ge } \\
\text { exposure }[\mathrm{kg} \cdot \mathrm{yr}]\end{array}$ & $\begin{array}{c}\text { active }{ }^{76} \mathrm{Ge} \\
\text { exposure }[\mathrm{kg} \cdot \mathrm{yr}]\end{array}$ \\
\hline M1-enrBEGe & $29{ }^{\text {enr } \mathrm{BEGe}^{\dagger}}$ & $19.362 \pm 0.029$ & $15.06 \pm 0.40$ & $32.124 \pm 0.048$ & $25.08 \pm 0.45$ \\
M1-enrCoax & $7{ }^{\text {enr Coax }}$ & $15.576 \pm 0.007$ & $11.61 \pm 0.54$ & $28.088 \pm 0.013$ & $21.0 \pm 1.0$ \\
M2-enrGe & all enriched & $34.938 \pm 0.030$ & $26.67 \pm 0.67$ & $60.212 \pm 0.050$ & $46.1 \pm 1.1$ \\
\hline
\end{tabular}

$\dagger$ The BEGe detector GD02D is the only detector that does not fully deplete [13]. Hence, events triggered by this detector are not considered in either data set. GD02D is omitted from the mass computation.

Table 1. Properties of the data sets considered in this analysis. Further details about the GerDA detectors can be found in past publications $[13,14]$.

tion are used only to determine the event multiplicity but do not enter any data set, e.g. an event that triggers three detectors one of which cannot be calibrated well is not considered a two- but a three-detector event. Also, two-detector events involving a detector which is not well calibrated are rejected. Events with a multiplicity higher than two are discarded by default and, likewise, events which trigger the muon veto are excluded.

\subsection{Analysis data sets}

Events of multiplicity one (M1) and multiplicity two (M2) from detectors with enriched isotope composition are accounted for in the construction of the analysis data sets. Events from the coaxial detectors with natural isotope composition, located in the central detector string, are not used in this analysis due to large uncertainties on their $\mathrm{n}^{+}$contact thickness and detection efficiency. The M1 events are split in two data sets based on the two enriched detector geometries which we call M1-enrBEGe and M1-enrCoax in the following. The M2 data form a third data set which is named M2-enrGe. The energy we associate to an M2 event is the sum of the energies reconstructed in the two detectors. The data sets, their exposure and respective detector mass are listed in table 1.

\subsection{Monte Carlo simulations and probability density functions}

The Probability Density Functions (PDFs) used to model contributions to the energy spectra are obtained from Monte Carlo simulations. The latter are performed using the MAGE simulation framework [18], based on GEANT4 v10.4 [19-21]. MAGE contains a software implementation of the GERDA Phase II detectors as well as the assembly and all other surrounding hardware components. A visualization of this implementation is presented in figure 1. Detector intrinsic $2 \nu \beta \beta$ decays of ${ }^{76} \mathrm{Ge}$ and background events originating from radioactive contaminations in and around the detector assembly are simulated. The energy spectrum of the two electrons emitted in the $2 \nu \beta \beta$ decay was sampled according to the distribution given in reference [22] implemented in DECAY0 [23]. The PDFs are obtained from the Monte Carlo simulations, taking into account the finite energy resolution and individual exposure acquired with each detector during the considered data taking periods. Special care is taken not to statistically bias the PDFs by assuring that each simulated decay is taken into account only once in the production of a PDF. For more details see appendix C. 
(e)

(f)

(a)

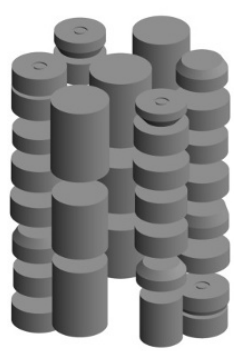

(c)

(b)

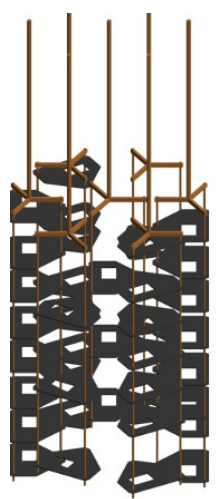

(d)

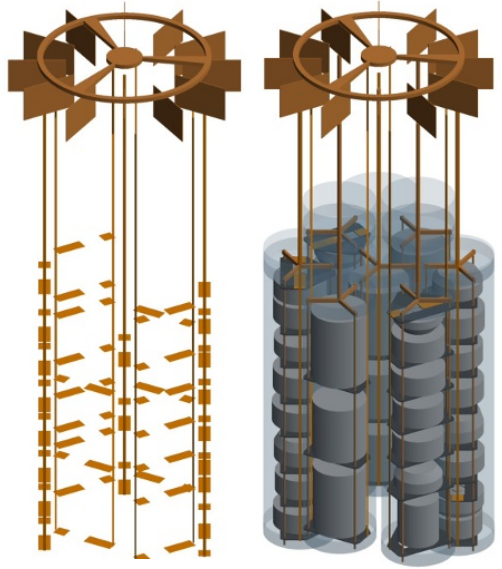

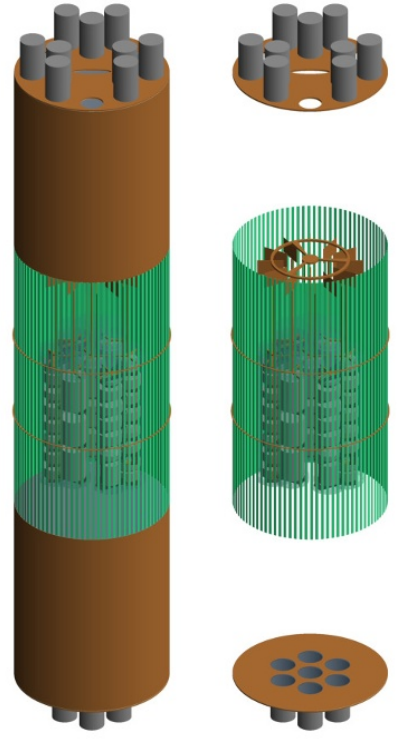

Figure 1. Implementation of the GERDA array in MAGE, visualized using the GEANT4 visualization drivers. From left to right: a) the GERDA detectors, b) the holder mounting, composed of silicon plates and copper bars c) the high-voltage and signal flexible flat cables plus the front-end electronics on top, d) the full array instrumentation, including the transparent nylon mini-shrouds, e) the full LAr veto system surrounding the array, including the fiber shroud (in green), the Tetratex ${ }^{\circledR}$ coated copper shrouds (above and below the fibers) and the two PMT arrays, f) the LAr veto system without the copper shrouds.

\subsection{Background expectation}

The event energy distribution of the three data sets is displayed in figure 2 ; the sum spectrum of M1-enrBEGe and M1-enrCoax in the top panel and M2-enrGe in the bottom panel. For the single-detector data, in the top panel, the following features are most noticeable: the $\beta$ decay of ${ }^{39} \mathrm{Ar}$ dominates the spectrum up to $565 \mathrm{keV}$ while between 600 and $1500 \mathrm{keV}$ the most prominent component is the continuous spectrum of $2 \nu \beta \beta$ decay of ${ }^{76} \mathrm{Ge}$. Two $\gamma$ lines at 1461 and $1525 \mathrm{keV}$ can be attributed to ${ }^{40} \mathrm{~K}$ and ${ }^{42} \mathrm{~K}$; further visible $\gamma$ lines belonging to ${ }^{85} \mathrm{Kr},{ }^{208} \mathrm{Tl},{ }^{214} \mathrm{Bi}$ and ${ }^{228} \mathrm{Ac}$ are indicated in the figure. The highest energies displayed are dominated by a peak like structure emerging at $5.3 \mathrm{MeV}$ with a pronounced low energy tail. This is a typical spectral feature of $\alpha$ particles and can, here, be attributed to ${ }^{210} \mathrm{Po}$ decay on the thin detector $\mathrm{p}^{+}$surfaces [14]. Events above the ${ }^{210} \mathrm{Po}$ peak belong to $\alpha$ decays emerging from the ${ }^{226} \mathrm{Ra}$ sub-chain on the detector $\mathrm{p}^{+}$surfaces. All these components contribute also to M2-enrGe except for ${ }^{39} \mathrm{Ar}, 2 \nu \beta \beta$ and high energy $\alpha$ components. This is due to the short range of $\alpha$ (tens of $\mu \mathrm{m}$ ) and $\beta$ particles (typically smaller than $1.5 \mathrm{~cm}$ ) in LAr and germanium with respect to the distance between detectors which is of the order of several $\mathrm{cm}$. 


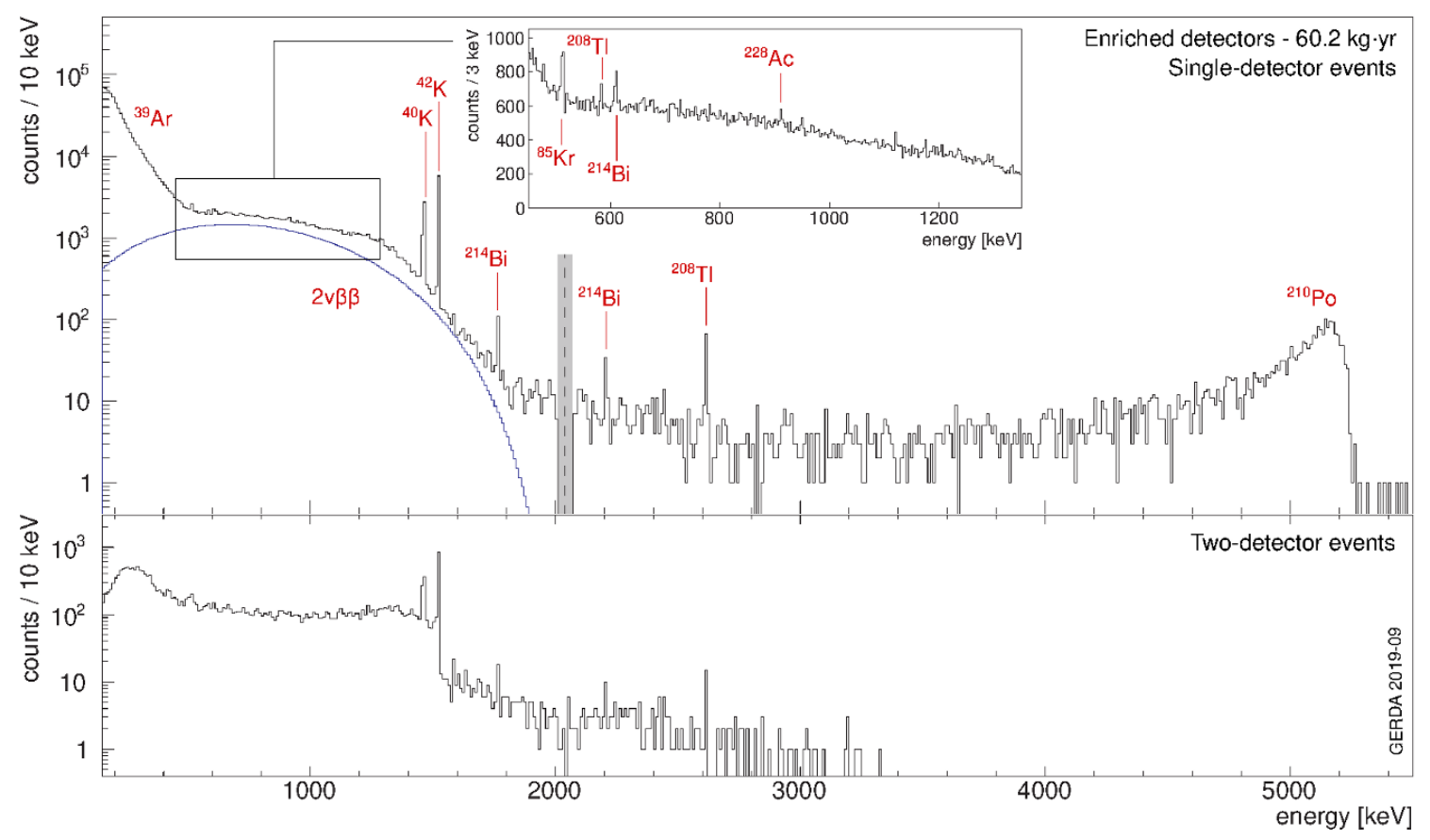

Figure 2. Summed energy spectra of single-detector events (M1-enrBEGe and M1-enrCoax, top panel) and two-detector events (M2-enrGe, bottom panel) collected in GERDA Phase II. The prominent features due to detector intrinsic $2 \nu \beta \beta$ events, ${ }^{42} \mathrm{~K},{ }^{39} \mathrm{Ar}$ and ${ }^{85} \mathrm{Kr}$ in the LAr, ${ }^{40} \mathrm{~K}$, the ${ }^{232} \mathrm{Th}$ and ${ }^{238} \mathrm{U}$ decay chains are highlighted. The window blinded for the $0 \nu \beta \beta$ analysis $\left(Q_{\beta \beta} \pm 25 \mathrm{keV}\right)$ is marked in grey.

The structural components of the setup have been screened for their radio-purity before deployment. Two measurement methods were used depending on the screened isotope: $\gamma$ ray spectroscopy $(\mathrm{Ge}-\gamma)$ with High Purity Germanium (in four underground laboratories, for details see reference [4]) and mass spectrometry with Inductively Coupled Plasma Mass Spectrometers (ICP-MS) [24]. Especially materials close to the detectors have been screened for radioactive contaminations originating from the ${ }^{238} \mathrm{U}$ and ${ }^{232} \mathrm{Th}$ decay chains, ${ }^{40} \mathrm{~K}$ and ${ }^{60} \mathrm{Co}$. For measured specific activities and upper limits see reference [5] section 5. All possible background sources taken into consideration in this analysis are described in detail below. The descriptions are accompanied by a selection of PDFs in figure 3 (see also appendix C).

${ }^{232} \mathrm{Th}$ and ${ }^{238} \mathbf{U}$ decay chains. The only isotopes simulated are ${ }^{234 \mathrm{~m}} \mathrm{~Pa},{ }^{214} \mathrm{~Pb}$ and ${ }^{214} \mathrm{Bi}$ from the ${ }^{238} \mathrm{U}$ decay chain and ${ }^{228} \mathrm{Ac},{ }^{212} \mathrm{Bi}$ and ${ }^{208} \mathrm{Tl}$ from the ${ }^{232} \mathrm{Th}$ decay chain. The following groups of isotopes are assumed to be in secular equilibrium: $\left[{ }^{238} \mathrm{U},{ }^{234 \mathrm{~m}} \mathrm{~Pa}\right]$ $\left[{ }^{226} \mathrm{Ra},{ }^{214} \mathrm{~Pb},{ }^{214} \mathrm{Bi}\right]\left[{ }^{228} \mathrm{Ra},{ }^{228} \mathrm{Ac}\right]$ and $\left[{ }^{228} \mathrm{Th},{ }^{212} \mathrm{Bi},{ }^{208} \mathrm{Tl}\right]$. Their decay products consist of $\gamma$ or $\beta$ particles with an energy higher than $520 \mathrm{keV}$. Less energetic particles from the remaining constituents in the chain do not enter the energy window which is considered in the presented analysis. The $\alpha$ emitters from the decay chains contaminating the thin $\mathrm{p}^{+}$ electrodes are described below. 
${ }^{60}$ Co. A significant fraction of components in the GERDA setup is made of copper [5], which can be produced with high radio-purity but is potentially activated by cosmic rays producing the long-lived isotope ${ }^{60}$ Co. The latter decays with a half-life of 5.2711(8) yr; from material screening it is also expected to be found in some of the detector high-voltage flexible flat cables.

${ }^{40} \mathbf{K}$. This isotope is found in all screened materials. Construction materials were not optimized for ultra-low ${ }^{40} \mathrm{~K}$ content because the Q-value of its decay is well below $Q_{\beta \beta}$ and hence does not contribute to the background in the ROI. The ${ }^{40} \mathrm{~K}$ decay spectrum exhibits a $\gamma$ line at $1460.822(6) \mathrm{keV}$ with an accumulated statistics on the order of $100 \mathrm{cts} /$ detector. In figure 12 the expected counts per detector for ${ }^{40} \mathrm{~K}$ simulated in different locations are shown. Using the ratio of events detected in different detectors, information about the spatial distribution of ${ }^{40} \mathrm{~K}$ can be extracted. We use this spatial information to resolve degeneracies of ${ }^{40} \mathrm{~K}$ in the energy spectra (for details see appendix $\mathrm{A}$ ).

${ }^{42} \mathbf{K}$. A cosmogenically produced isotope in LAr is ${ }^{42} \operatorname{Ar}\left(T_{1 / 2}=32.9(11)\right.$ yr $)$ which decays to ${ }^{42} \mathrm{~K}$. The distribution of ${ }^{42} \mathrm{~K}$ inside the LAr is likely to be inhomogeneous due to drift of the ionized decay products induced by the electric field (generated by high-voltage cables and detectors) and convection. ${ }^{42} \mathrm{~K}$ decays to ${ }^{42} \mathrm{Ca}$ via $\beta$ decay with a half-life of $12.355(7) \mathrm{h}$ and a Q-value of 3525.22(18) keV, well above $Q_{\beta \beta}$. For the $\beta$ particle to be detected the decay needs to happen within a distance of a few centimeters ${ }^{1}$ to the detector surface. As the detectors are in direct contact with the LAr, the $\beta$ component of ${ }^{42} \mathrm{~K}$ potentially gives one of the most significant contributions to the background in the ROI. Therefore, we separate decays originating inside and outside the mini-shrouds in the following analysis. The full-range fit has little sensitivity to any potassium inhomogeneity outside the minishrouds. Based on detector-wise observations, however, a surplus of ${ }^{42} \mathrm{~K}$ above the detector array in the vicinity of the front-end electronics is deduced (see appendix A). Outside the mini-shrouds we, hence, consider a homogeneous component and an additional distribution above the detector array. Inside the mini-shrouds the $\beta$ spectrum becomes potentially important. Some scenarios are possible, the closer ${ }^{42} \mathrm{~K}$ decays to the detector surface, namely to the $\mathrm{n}^{+}$and $\mathrm{p}^{+}$contacts, the more $\beta$ particles enter the germanium. A fraction of events around $Q_{\beta \beta}$ coming from ${ }^{42} \mathrm{~K}$ is potentially due to $\gamma$ particles with higher energy and sub-percent level branching ratio or simultaneous energy deposition of multiple $\gamma$ particles. This $\gamma$ component could become important for large quantities of ${ }^{42} \mathrm{~K}$ not located directly on the detector surfaces with the $\beta$ particle being absorbed in the LAr. As for ${ }^{40} \mathrm{~K}$ also the $\gamma$ line at $1525 \mathrm{keV}$ of ${ }^{42} \mathrm{~K}$ contains valuable information about the spatial decay distribution of this isotope. In contrast to ${ }^{40} \mathrm{~K}$ no additional information, e.g. from radiopurity screening measurements, is available. For more detailed information about ${ }^{40} \mathrm{~K}$ and ${ }^{42} \mathrm{~K}$ see appendix A.

\footnotetext{
${ }^{1}$ The path length of ${ }^{42} \mathrm{~K} \beta$ particles in LAr is less than $1.6 \mathrm{~cm}$, but bremsstrahlung photons from the interaction with LAr can travel as far as $\sim 10 \mathrm{~cm}$.
} 
$\boldsymbol{\alpha}$ emitters. The lithium-diffused $\mathrm{n}^{+}$detector surfaces act as a barrier for $\alpha$ particles. The latter can only penetrate the very thin boron-implanted $\mathrm{p}^{+}$-contact or the contact separating groove. $\alpha$ particles have to be emitted directly at the surface or from a thin adjacent layer of LAr. Since $\alpha$ particles have to cross the $\sim 0.5 \mu \mathrm{m}$ thick $\mathrm{p}^{+}$dead layer and therefore only part of their initial energy is deposited in the active volume, this background component leads to peaks with characteristic low-energy tails in the HPGe energy spectra (see figure 3e). Some $\alpha$ events, presumably originating from the detector groove, are reconstructed with degraded energy and lead to an additional, continuous spectral component. We find mainly ${ }^{210} \mathrm{Po}$ but also traces of isotopes from the ${ }^{226} \mathrm{Ra}$ decay chain.

Detector bulk impurities. Cosmogenically produced long-lived isotopes can also be found in germanium [25-27]. In particular, ${ }^{68} \mathrm{Ge}$ and ${ }^{60} \mathrm{Co}$ can occur as detector intrinsic impurities with half-lives of 270.93(13) d and 5.2711(8) yr. The BEGe detectors were kept underground during major parts of the fabrication and characterization operations. Periods when these detectors were above ground have been tracked in a database [12]. Thus, for the well-monitored BEGe detectors we expect impurities of 5 nuclei $/ \mathrm{kg}$ of ${ }^{68} \mathrm{Ge}$ and 21 nuclei $/ \mathrm{kg}$ of ${ }^{60} \mathrm{Co}$ as of September 2014 [12]. Extrapolating the expected impurities to the whole Phase II data taking period we expect on average $0.03 \mathrm{cts} /$ day from ${ }^{68} \mathrm{Ge}$ and $0.1 \mathrm{cts} /$ day due to ${ }^{60}$ Co. From background modeling in Phase I [14] the contribution for the coaxial detectors formerly used in the Heidelberg-Moscow (HDM) [28] and IGEx [29] experiments is expected to be even smaller due to their long storage underground. Simulating the expected detector bulk impurities we find background contributions around $Q_{\beta \beta}$ of less than $10^{-4} \mathrm{cts} /(\mathrm{keV} \cdot \mathrm{kg} \cdot \mathrm{yr})$ in both cases. Hence, we conclude that ${ }^{68} \mathrm{Ge}$ as well as ${ }^{60} \mathrm{Co}$ can be neglected in the following analysis. Potential bulk contaminations with ${ }^{238} \mathrm{U}$ and ${ }^{232} \mathrm{Th}$ were studied in reference [30]. Only upper limits were found, establishing germanium crystals as material of outstanding radio-purity. Hence, we only consider the decay of ${ }^{76} \mathrm{Ge}$ via $2 \nu \beta \beta$ as detector intrinsic background component while all other intrinsic impurities are considered to be negligible.

Other sources. As discussed in reference [14], prompt cosmic muon induced background events are efficiently vetoed by the identification of Cerenkov light emitted by muons when they pass the water tank. The expected BIs, due to the direct muon and neutron fluxes at the LNGS underground laboratory, have been estimated to be of the order $3 \cdot 10^{-5} \mathrm{cts} /(\mathrm{keV} \cdot \mathrm{kg} \cdot \mathrm{yr})[31]$ and $10^{-5} \mathrm{cts} /(\mathrm{keV} \cdot \mathrm{kg} \cdot \mathrm{yr})$ [27] in earlier works, respectively. Background contributions coming from delayed decays of ${ }^{77} \mathrm{Ge}$ and ${ }^{77 \mathrm{~m}} \mathrm{Ge}$, also induced by cosmic muons, are estimated to be $0.21 \pm 0.01$ nuclei/(kg.yr) [32] corresponding to a BI prior to the active background suppression techniques of about $10^{-5} \mathrm{cts} /(\mathrm{keV} \cdot \mathrm{kg} \cdot \mathrm{yr})$. Also, the water tank and LAr cryostat contaminations are expected to contribute to the GERDA BI with less than $10^{-4} \mathrm{cts} /(\mathrm{keV} \cdot \mathrm{kg} \cdot \mathrm{yr})[4,33]$. All above mentioned contributions are considered negligible in this work. Other potential sources of background from interactions of ${ }^{76} \mathrm{Ge}[15,27]$ and ${ }^{206} \mathrm{~Pb}[34]$ with neutrons and ${ }^{56} \mathrm{Co}$ for which no evidence was found are not taken into consideration. The cosmogenically produced isotope ${ }^{39} \mathrm{Ar}$ and the anthropogenic isotope ${ }^{85} \mathrm{Kr}$ [35], which are dissolved in LAr, emit particles which are dominantly less energetic than the energy window which is considered in the presented analysis. 


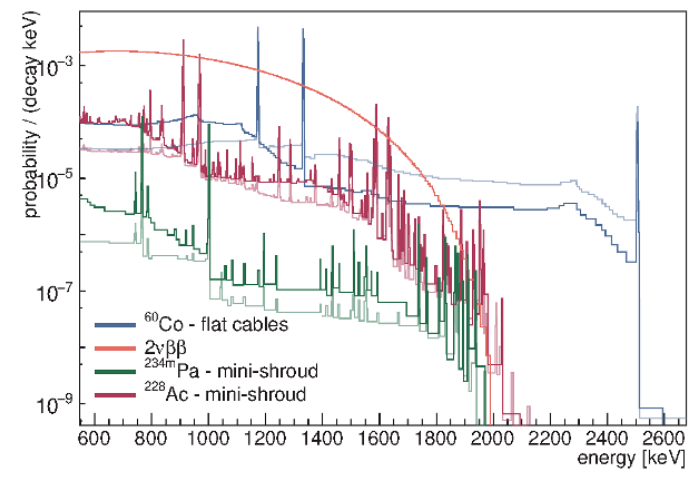

(a) ${ }^{60} \mathrm{Co},{ }^{234 \mathrm{~m}} \mathrm{~Pa},{ }^{228} \mathrm{Ac}$ contaminations and detector intrinsic $2 \nu \beta \beta$ decay.

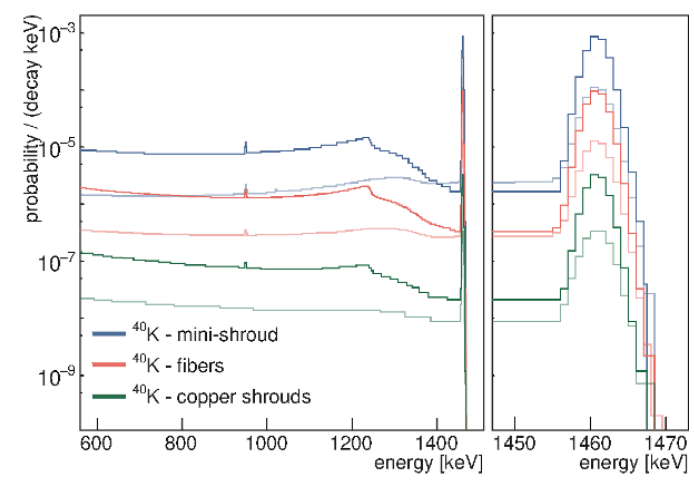

(c) ${ }^{40} \mathrm{~K}$ contamination close to the detector array (on the mini-shrouds), at a higher radial distance (on the fiber shroud) and higher vertical distance (on the copper shrouds).

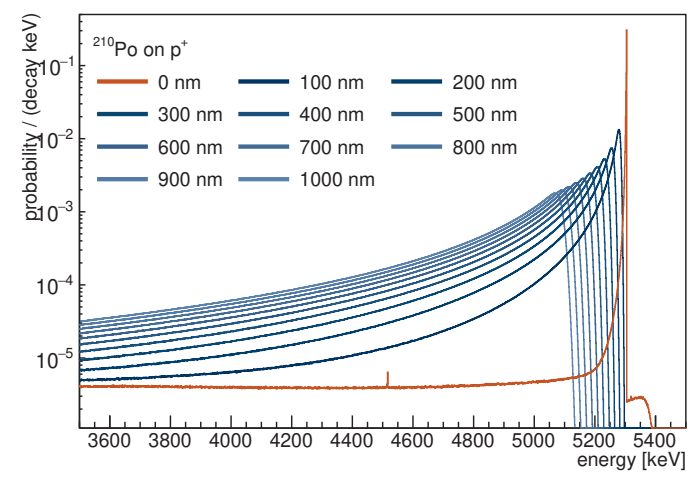

(e) ${ }^{210}$ Po $\alpha$ decays on $\mathrm{p}^{+}$contact surface for different thicknesses of the inactive contact layer. For $0 \mathrm{~nm}$ the nuclear recoil energy can be absorbed and some energy can be lost in the LAr.

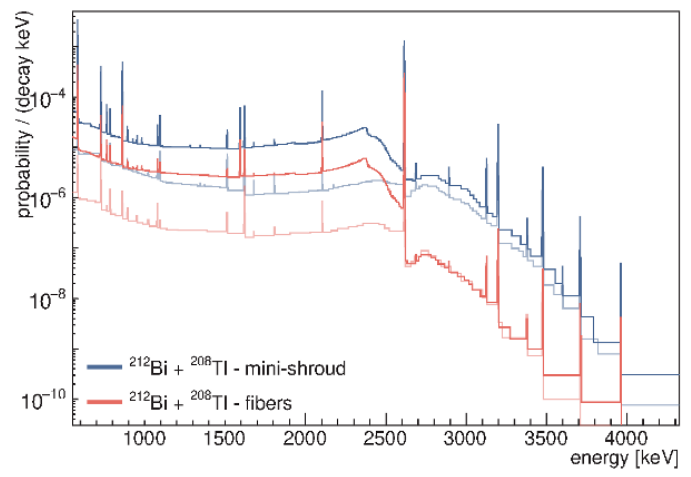

(b) ${ }^{212} \mathrm{Bi}$ and ${ }^{208} \mathrm{Tl}\left({ }^{232} \mathrm{Th}\right.$ chain) contaminations far from (fiber shroud) and close to (minishrouds) the detector array.

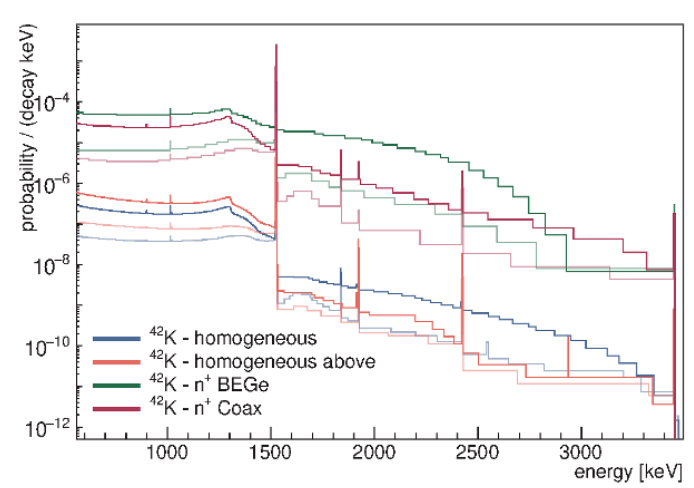

(d) ${ }^{42} \mathrm{~K}$ contamination in different locations inside the LAr.

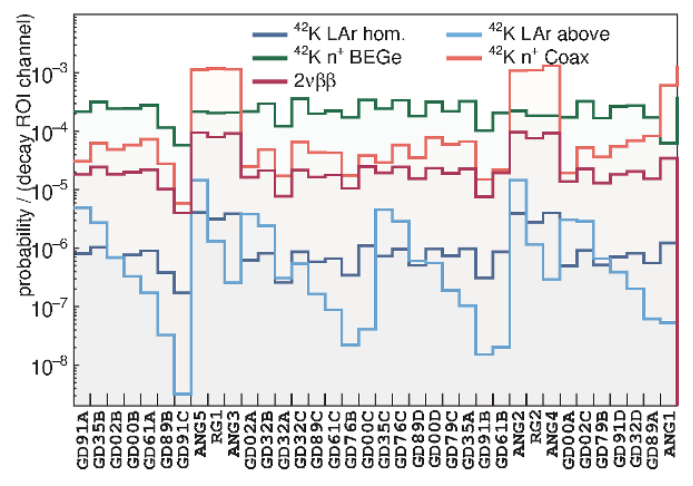

(f) ${ }^{42} \mathrm{~K}$ contamination in different volumes in the LAr and detector intrinsic $2 \nu \beta \beta$ for comparison. The energy window (ROI) considered is $(1525 \pm 4) \mathrm{keV}\left({ }^{42} \mathrm{~K} \gamma\right.$ line $)$.

Figure 3. From (a) to (e): PDFs in the full energy domain. The PDFs for the M1-enrGe (M1-enrBEGe + M1-enrCoax) (in fully opaque colors) and the M2-enrGe (in shaded colors) data sets relative to different background sources. For visualization purposes a variable binning is adopted. (f) PDFs per detector for the ${ }^{42} \mathrm{~K} \gamma$ line. All PDFs are normalized to the number of simulated primary decays. 


\section{Statistical analysis}

The multivariate statistical analysis, which is used to model and disentangle the background in its components, runs on the three binned data sets M1-enrBEGe, M1-enrCoax and M2-enrGe. It is based on the reconstructed energy with the zero area cusp (ZAC) filter algorithm which is close to optimal and provides an excellent low-frequency rejection [16]. The single-detector data sets M1-enrBEGe and M1-enrCoax contain the reconstructed ZAC energy of all M1 events whereas for the two-detector events the sum of the two reconstructed energies is put in the M2-enrGe data set. Moreover, the count rate per detector is used for the two potassium $\gamma$ lines. The spatial event distribution is a collection of the number of events per detector for M1 events and expressed in a matrix of pairs of detectors for all M2 events.

Assuming that the number of events in each bin follows the Poisson probability distribution Pois $(n ; \nu)$, where $\nu$ is the expected mean and $n$ is the experimentally measured number of counts, the likelihood function for a binned data set reads $\prod_{i=1}^{N_{\text {bins }}} \operatorname{Pois}\left(n_{i} ; \nu_{i}\right)$. Here $\nu_{i}=\sum_{k=1}^{N_{\text {com }}} \nu_{i}^{(k)}$ is the expected number of events in the $i$-th bin, calculated as the sum of the contributions from each background component $k ; \nu_{i}\left(\lambda_{1}, \ldots, \lambda_{N_{\mathrm{com}}}\right)$ is a function of the parameters of interests $\lambda_{j}$ (isotope activities, $2 \nu \beta \beta$ half-life, etc.). The complete likelihood function adopted for the present analysis combines the three data sets M1-enrBEGe, M1-enrCoax and M2-enrGe:

$$
\mathcal{L}\left(\lambda_{1}, \ldots, \lambda_{m} \mid \text { data }\right)=\prod_{d=1}^{N_{\text {dat }}} \prod_{i=1}^{N_{\text {bins }}} \operatorname{Pois}\left(n_{d, i} ; \nu_{d, i}\right) .
$$

The statistical inference is made within a Bayesian framework. Hence, to obtain posterior probabilities for the free parameters of interest $\lambda_{j}$, the likelihood defined in equation (3.1) is multiplied according to the Bayes theorem by a factor modeling the prior knowledge of each background component as presented in section 2.3. The computation is performed using a Markov Chain Monte Carlo (MCMC) and is implemented using the BAT software suite [36, 37]. Posterior probability distributions of any observable that is not a free parameter of the likelihood function, like background index estimates, are obtained by sampling the desired parameter from the MCMC. A $p$-value estimate is provided as a goodness-of-fit measure by adopting the algorithm suggested in reference [38] for Poissondistributed data. It has to be kept in mind that this $p$-value estimate, however, is not as well suited for model comparison as is for instance a Bayes factor; e.g. the number of free parameters is not taken into account while a Bayes factor always penalizes models that add extra complexity without being required by the data.

\subsection{Analysis window and binning}

The fit range and data bins are chosen such as to exploit as much information from spectral features as possible brought by data without introducing undesired bias. The chosen fit range in energy space for the single-detector data sets (M1-enrBEGe and M1-enrCoax) starts from just above the end-point of the ${ }^{39} \mathrm{Ar} \beta^{-}$spectrum at $565 \mathrm{keV}$ and ends just above the ${ }^{210} \mathrm{Po}$ peak at $5260 \mathrm{keV}$, where the event rate drops to almost zero values. For the 
two-detector events (M2-enrGe data set) the fit range starts at $520 \mathrm{keV}$ and extends up to $3500 \mathrm{keV}$. Possible additional components outside of this range (e.g. ${ }^{39} \mathrm{Ar}$ ) do neither add information to the background decomposition in the ROI around $Q_{\beta \beta}$ nor to the analysis of $2 \nu \beta \beta$ decay. Furthermore, at energies lower than $\sim 100 \mathrm{keV}$ the shape of the PDFs is dominated by uncertainties on the detector transition layer model, which describes the charge-carrier collection at the interface between the $\mathrm{n}^{+}$contact and the detector active volume. The exact nature of this transition region is different for each detector and prone to systematic uncertainties [39].

With an energy resolution which is typically $3-4 \mathrm{keV}$ at $Q_{\beta \beta}$ (FWHM) $[8,9]$ and better at lower energies, a fixed bin size of $1 \mathrm{keV}$ was chosen for all data sets. The only exceptions are the two $\gamma$ lines from ${ }^{40} \mathrm{~K}$ and ${ }^{42} \mathrm{~K}$ each of which is combined in a single bin from $1455 \mathrm{keV}$ to $1465 \mathrm{keV}$ and from $1520 \mathrm{keV}$ to $1530 \mathrm{keV}$, respectively. This is done in order to suppress any systematic uncertainties of the energy calibration and resolution model that affect the position and shape of the $\gamma$ lines [9].

\subsection{Likelihood factorization}

A feature of the selected data is that the likelihood in equation (3.1) can be factorized in uncorrelated parts which can be studied individually and in detail. In the following we shortly outline the parts of the data which were studied in depth based on the approach of factorizing the likelihood into uncorrelated parts. Finally, the results of these analyses are incorporated into a full-range fit. This procedure is equivalent to a simultaneous analysis of all data but increases the input knowledge for the fit and breaks down the computational complexity in smaller steps.

Potassium tracking analysis. As can be noted from figure $3 \mathrm{c}$ and figure $3 \mathrm{~d}$ the PDFs of ${ }^{40} \mathrm{~K}$ and ${ }^{42} \mathrm{~K}$ in energy are prone to degeneracies and hence parameter correlations. Their most prominent $\gamma$ lines at 1461 and $1525 \mathrm{keV}$, respectively, contain information on the spatial distribution while the two-detector events contain information about the angular distribution of Compton scattered events. Their combination is beneficial in order to pin down the potential location of the two potassium isotopes. In total the M1 data contains $4472 \mathrm{cts}$ in $1461 \pm 4 \mathrm{keV}$ and $6718 \mathrm{cts}$ in $1525 \pm 4 \mathrm{keV}$ while the M2 events contain $554 \mathrm{cts}$ in $1461 \pm 6 \mathrm{keV}$ and $865 \mathrm{cts}$ in $1525 \pm 6 \mathrm{keV}$, respectively. An analysis of the number of events in the two potassium $\gamma$ lines in each detector (and detector pair) is used to exploit mainly top-down and rotational asymmetries in the ${ }^{40} \mathrm{~K}$ and ${ }^{42} \mathrm{~K}$ distributions. The number of events in the two energy windows are summarized detector-by-detector; in the following we refer to this procedure as projection in detector space. The treatment of the likelihood in equation (3.1) is outlined in detail in appendix A. The number of events in all other $\gamma$ lines is too low in order to adopt a useful detector-wise analysis. The spatial analysis of ${ }^{40} \mathrm{~K}$ and ${ }^{42} \mathrm{~K}$ is incorporated in the full-range fit by directly employing the posterior parameter distributions as prior information. ${ }^{2}$

\footnotetext{
${ }^{2}$ By adopting this approach, a part of the data in the potassium $\gamma$ lines region is analyzed twice: first in the potassium tracking analysis and then in the full-range fit. Nevertheless, considering that the two analyses exploit different data features (i.e. count rate per detector and total count rate per energy) and the overlap between the two data set is minimal, the overall effect is negligible.
} 
$\alpha$ events background analysis. The single-detector energy spectra above $3.5 \mathrm{MeV}$ (the $\mathrm{Q}$-value of ${ }^{42} \mathrm{~K} \beta$ decay) are strongly dominated by $\alpha$ events. They are not present in twodetector data due to the short range of $\alpha$ particles in LAr and germanium. Also, this component is not correlated to other backgrounds considered here because it peaks at energies well above the highest $\gamma$ emission energies and $\beta$ decay Q-values. A careful study was carried out considering various $\mathrm{p}^{+}$contact thickness and event rates to reproduce the ${ }^{210}$ Po peak. In order to reproduce $\alpha$ events with degraded energy an empirical model is fit to the data. A linear function with free slope and offset and a cut-off below the maximum of the ${ }^{210} \mathrm{Po}$ peak fits the data well. The agreement of the $\alpha$ background model with the data is demonstrated in appendix B and figure 9 therein. Information from the detailed analysis of the high-energy $\alpha$ region is incorporated in the full-range fit using a combined PDF that summarizes the ${ }^{210} \mathrm{Po}$ peak plus the ${ }^{226} \mathrm{Ra}$ decay chain and a linear floating component for degraded $\alpha$ events.

\subsection{Prior distributions}

The following criteria are adopted to convert the prior information described in section 2.3 into prior probability distributions on the parameters of interest: ${ }^{3}$ if a measured value with uncertainty is available for a background contamination then a Gaussian distribution with a corresponding centroid and a $1 \sigma$ width is adopted. In presence of a $90 \%$ C.L. upper limit, instead, an exponential prior distribution is constructed with $90 \%$ of its area covering parameter values from 0 up to the given 90 \% C.L. upper limit. A uniform prior distribution is assigned to components for which no measured value or upper limit is available. Ranges for uniform priors are initially taken very wide, in order to span a large portion of the allowed parameter space, then optimized to contain at least $99 \%$ of the posterior distribution. As mentioned before, in addition to the information from screening measurements, prior distributions for ${ }^{40} \mathrm{~K}$ and ${ }^{42} \mathrm{~K}$ are constructed considering the posterior inference from their spatial distribution. ${ }^{4}$ Moreover, as ${ }^{214} \mathrm{Bi}$ is part of the ${ }^{226} \mathrm{Ra}$ decay chain, we constrain a ${ }^{214} \mathrm{Bi}$ component on the $\mathrm{p}^{+}$contact by a Gaussian prior extracted from the obtained ${ }^{226} \mathrm{Ra}$ activity based on the energy estimator in the high-energy $\alpha$ region.

\section{Results}

As described in section 3.2 the $\alpha$ event background and potassium $\gamma$ lines are studied individually and the results are incorporated in the full-range fit as prior distributions. The latter combines a simultaneous fit of the M1 and the M2 data sets. For the final combination of parameters, outlined in this section, components with a posterior distribution peaked at zero were eliminated from the fit. The stability of the results with respect to the bin size and prior distributions was verified. Changing the prior distribution for fit parameters for which no screening measurement is available from a flat to an exponential one does not

\footnotetext{
${ }^{3}$ In Bayesian analysis the prior probability distribution describes all knowledge about an unobserved quantity of ultimate interest before taking the data into account.

${ }^{4}$ The Bayesian posterior distribution is the conditional probability distribution of the unobserved quantities of ultimate interest, given the observed data.
} 
significantly impact the final posterior distributions. The compatibility of the final model, which includes 34 free fit parameters, with data is supported by a $p$-value of $\sim 0.3$.

The estimated activities of individual components and other parameters of interest are listed in table 2. In particular, for each component we report the global and the marginalized mode of the posterior parameter distribution, along with its smallest $68 \%$ C.I. The global mode corresponds to the global best fit value while the marginalized mode is the most probable parameter value when integrating over all other parameters. The original type of prior distribution is marked with [f] for flat, [g] for Gaussian and [e] for exponential; the latter two are used if screening measurements are available. Subsequently, for all ${ }^{40} \mathrm{~K}$ and ${ }^{42} \mathrm{~K}$ components, the prior distribution is imported from the potassium tracking analysis and for ${ }^{214} \mathrm{~Pb}$ and ${ }^{214} \mathrm{Bi}$ on the $\mathrm{p}^{+}$contact from the reconstructed ${ }^{226} \mathrm{Ra}$ content from the $\alpha$ events background analysis.

The spectral decomposition of all data sets is shown in figure 4. For each data set the residual distribution as a multiple of the expected $1 \sigma$ fluctuation in each bin is displayed. We find for the M1-enrBEGe data set $66.4 \%, 94.5 \%$ and $99.6 \%$ of points in the $1 \sigma-, 2 \sigma-$ and $3 \sigma$-bands, for the M1-enrCoax data set $66.0 \%, 94.7 \%$ and $99.8 \%$ and for the M2-enrGe data set $70.0 \%, 96.1 \%$ and $99.7 \%$, respectively. Thus, in all three cases the residuals are normally distributed. No outliers with residuals larger than $3 \sigma$ are found in a $\pm 50 \mathrm{keV}$ window around $Q_{\beta \beta}$ and the bins exceeding $3 \sigma$ do not correspond to any noted $\gamma$ line.

The ${ }^{42} \mathrm{~K}$ distribution is optimized to best fit the data. In order to disentangle the ${ }^{42} \mathrm{~K}$ $\gamma$ and $\beta$ components, the volume inside and outside of the mini-shrouds is separated in the PDF construction. Inside the mini-shrouds a homogeneous distribution is compatible with the data as well as ${ }^{42} \mathrm{~K}$ attached to the detectors contact surfaces. In the fit model given here, a possible scenario is chosen where all ${ }^{42} \mathrm{~K}$ is located on the $\mathrm{n}^{+}$surfaces. However, we note that ${ }^{42} \mathrm{~K}$ on the $\mathrm{p}^{+}$appears to partly substitute the energy-degraded $\alpha$ component in the M1-enrCoax data set if introduced in the fit and predicts a higher total BI around $Q_{\beta \beta}$. The extracted ${ }^{42} \mathrm{~K}$ activity on the ${ }^{\text {enr }}$ Coax $\mathrm{p}^{+}$contact in this case is $22 \pm 4 \mu \mathrm{Bq}$ corresponding to a contribution to the BI around $Q_{\beta \beta}$ of $(7 \pm 1) \cdot 10^{-3} \mathrm{cts} /(\mathrm{keV} \cdot \mathrm{kg} \cdot \mathrm{yr})$. For the M1-enrBEGe data set the posterior distribution of a possible ${ }^{42} \mathrm{~K}$ component on the $\mathrm{p}^{+}$ contact is compatible with zero. Outside the mini-shrouds an inhomogeneous distribution of the ${ }^{42} \mathrm{~K}$ decays explains the observations better. Detectors which are located at higher positions in the strings show an excess of events in the ${ }^{42} \mathrm{~K} 1525 \mathrm{keV} \gamma$ line which is compatible with a surplus of ${ }^{42} \mathrm{~K}$ located right above the detector array (see appendix $\mathrm{A}$ ). The full-range fit model contains a homogeneous ${ }^{42} \mathrm{~K}$ distribution outside the mini-shrouds which is reconstructed with a specific activity of $186 \pm 39 \mu \mathrm{Bq} / \mathrm{kg}$ plus an additional distribution in the vicinity of the cables above the array.

A large fraction of the contamination with ${ }^{40} \mathrm{~K}$ in the setup cannot be accounted for by the screened hardware listed in table 2 . We thus add a close $(\sim 1 \mathrm{~cm})$ and a far $(\sim 50 \mathrm{~cm})$ ${ }^{40} \mathrm{~K}$ component with respect to the detector array which are in fact replica of the PDFs for the mini-shrouds and the Tetratex ${ }^{\circledR}$-coated copper shrouds. These additional components absorb the excess indicated by the fit, the largest part of the reconstructed events in the spectra is attributed to impurities close to the array. 


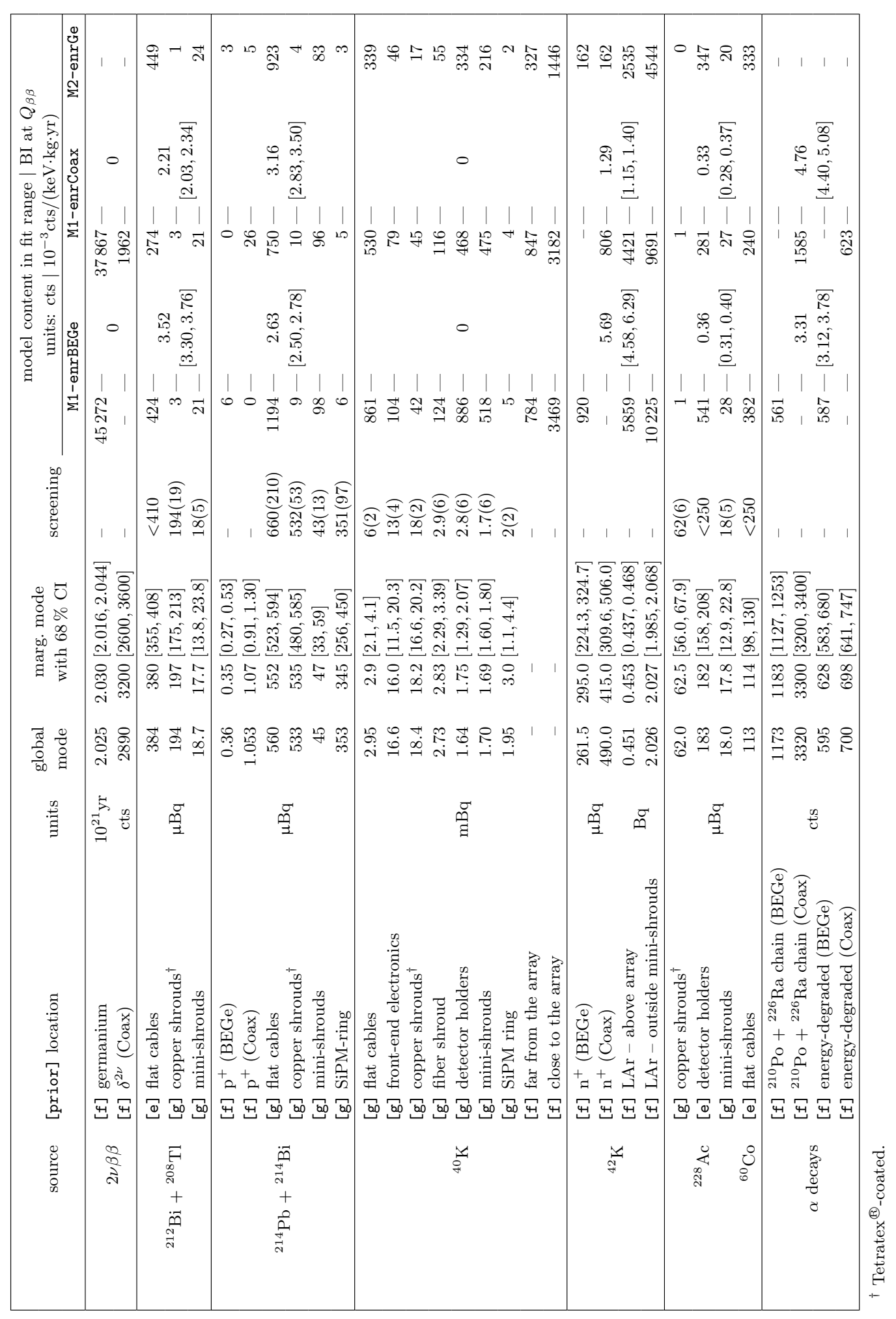

눈

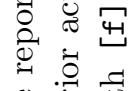

空总

$-10$

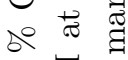

$\infty \overrightarrow{0}$.

若焉

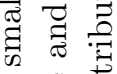

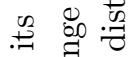

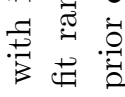

吅

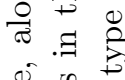

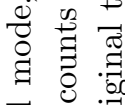

渮

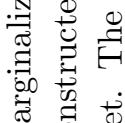

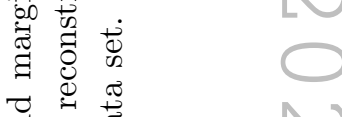

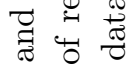

:

뜽 크

유ㅇㅛㅠ

نे

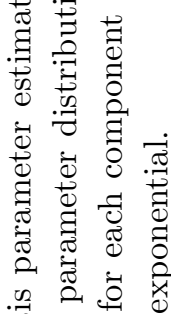

客

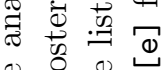
$\cong$ 은 范

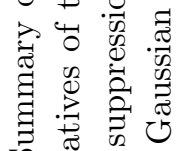

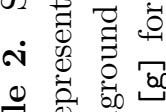



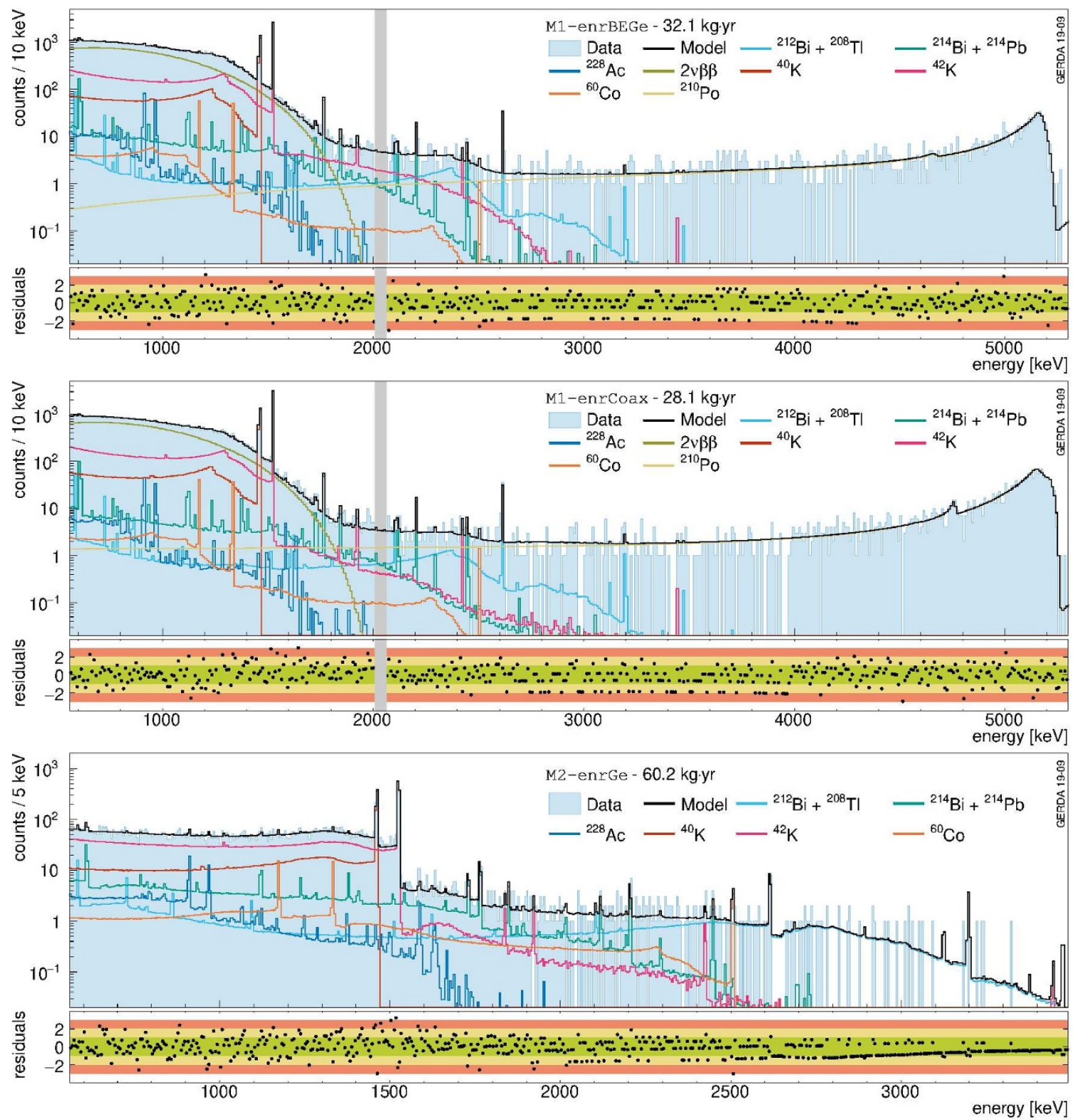

Figure 4. Background decomposition of the event energy distributions of the (from top to bottom) M1-enrBEGe, M1-enrCoax and M2-enrGe data sets. Components referring to the same background source in different locations are summed together for visualization convenience. The blinded region $Q_{\beta \beta} \pm 25 \mathrm{keV}$ is highlighted in gray. In the three lower panels displaying the normalized residual distributions the central $1 \sigma$-, $2 \sigma$ - and $3 \sigma$-bands are marked in green, yellow and red, respectively. Note that for bins with low expected statistics due to the discrete nature of the measured spectrum not all colored bands are meaningful [40].

The ${ }^{40} \mathrm{~K}$ and ${ }^{42} \mathrm{~K}$ distributions can be further split into smaller volumes and studied as an extension of the potassium tracking analysis (as described in section 3.2) projected in detector space. The additional ${ }^{40} \mathrm{~K}$ component close to the array and the ${ }^{42} \mathrm{~K}$ component above the array are split into 7 sub-components on a string-by-string basis. The potas- 
sium concentration is in general found to be asymmetric among the detector strings. In particular, a more prominent ${ }^{42} \mathrm{~K}$ concentration is found above the central string. This is consistent with the electrostatic drift of ${ }^{42} \mathrm{~K}$ ions induced by the electric field in the LAr which is generated by the unshielded high-voltage flat cables biased with about $4 \mathrm{kV}$. The ${ }^{40} \mathrm{~K}$ and ${ }^{42} \mathrm{~K}$ spatial analysis fitting the potassium $\gamma$ lines projected in detector space is presented in full detail in appendix A.

The $\alpha$ distribution is adjusted to best fit the data. The ${ }^{210} \mathrm{Po}$ peak at $5.2 \mathrm{MeV}$ is found to be best described by a mixture of PDFs obtained assuming different $\mathrm{p}^{+}$contact thicknesses confirming results of the Phase I background analysis [14]. The empirical linear model which is used to describe $\alpha$ events with degraded energy (see section 3.2), extends down to $Q_{\beta \beta}$ and below. For the M1-enrBEGe data set $\alpha$ events are efficiently isolated using pulse shape discrimination (PSD) techniques. The compatibility of the degraded-energy $\alpha$ component with $\alpha$ events identified by PSD was checked and is found consistent. All details about the $\alpha$ events analysis can be found in appendix B.

Smaller contributions to the background model in the full energy range are attributed to ${ }^{214} \mathrm{~Pb}$ and ${ }^{214} \mathrm{Bi}$ from the ${ }^{238} \mathrm{U}$ decay chain, ${ }^{228} \mathrm{Ac},{ }^{212} \mathrm{Bi}$ and ${ }^{208} \mathrm{Tl}$ from the ${ }^{232} \mathrm{Th}$ decay chains and ${ }^{60} \mathrm{Co}$. With a total contribution in the fit range of $10^{-3} \mathrm{cts} / \mathrm{keV}$ for both the M1-enrBEGe and M1-enrCoax data set ${ }^{234 \mathrm{~m}} \mathrm{~Pa}$ gives negligible contribution to the spectra and is therefore dropped from the full-range fit model. The central values preferred in the full-range fit are driven by screening measurements and the spectral contributions are all fully accounted for by the listed hardware components. The only exception is ${ }^{214} \mathrm{~Pb}$ and ${ }^{214} \mathrm{Bi}$ where a minor contribution is added on the $\mathrm{p}^{+}$contact expected from the observation of $\alpha$ events belonging to the ${ }^{226} \mathrm{Ra}$ decay chain.

Most counts in the fit range are attributed to the $2 \nu \beta \beta$ decay of ${ }^{76} \mathrm{Ge}$; in fact its continuous distribution dominates the spectrum up to almost $1.9 \mathrm{MeV}$. Here, we base the $2 \nu \beta \beta$ half-life estimate on the M1-enrBEGe data set only. An additional parameter, $\delta^{2 \nu}$, parameterizes the observed discrepancy to the value solely derived from the M1-enrCoax data set. The value of $\delta^{2 \nu}$ extracted from the fit amounts to a surplus of $5 \%$ of $2 \nu \beta \beta$ counts observed in M1-enrCoax. It mainly quantifies the systematic biases between the active

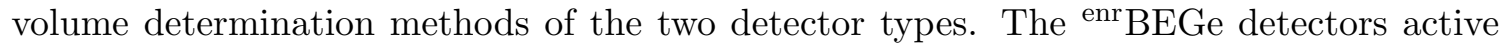
volume measurements are affected by a smaller systematic uncertainty than the ${ }^{\text {enr }}$ Coax detectors $[13,14]$. Hence, the extracted $2 \nu \beta \beta$ half-life, based on the M1-enrBEGe data set and given here only with statistical uncertainties, amounts to $T_{1 / 2}^{2 \nu}=(2.03 \pm 0.02) \cdot 10^{21} \mathrm{yr}$. A detailed discussion follows in section 5 .

The background model describes the individual contributions to the total BI around $Q_{\beta \beta}$ prior active background suppression (see figure 5). The BI is defined as the number of counts over exposure and energy in the energy window from $1930 \mathrm{keV}$ to $2190 \mathrm{keV}$ excluding the region around $Q_{\beta \beta}\left(Q_{\beta \beta} \pm 5 \mathrm{keV}\right)$ and the intervals $2104 \pm 5 \mathrm{keV}$ and $2119 \pm 5 \mathrm{keV}$, which correspond to known $\gamma$ lines from ${ }^{208} \mathrm{Tl}$ and ${ }^{214} \mathrm{Bi}$. The values for each background contribution are given in table 2. The dominating background contribution around $Q_{\beta \beta}$ in the M1-enrBEGe data set come from ${ }^{42} \mathrm{~K}$. Isotopes from the ${ }^{232} \mathrm{Th}$ decay chain, $\alpha$ particles mainly with degraded energy and isotopes from the ${ }^{238} \mathrm{U}$ decay chain contribute about equally. The estimated total BIs extracted from the marginalized posterior distributions 

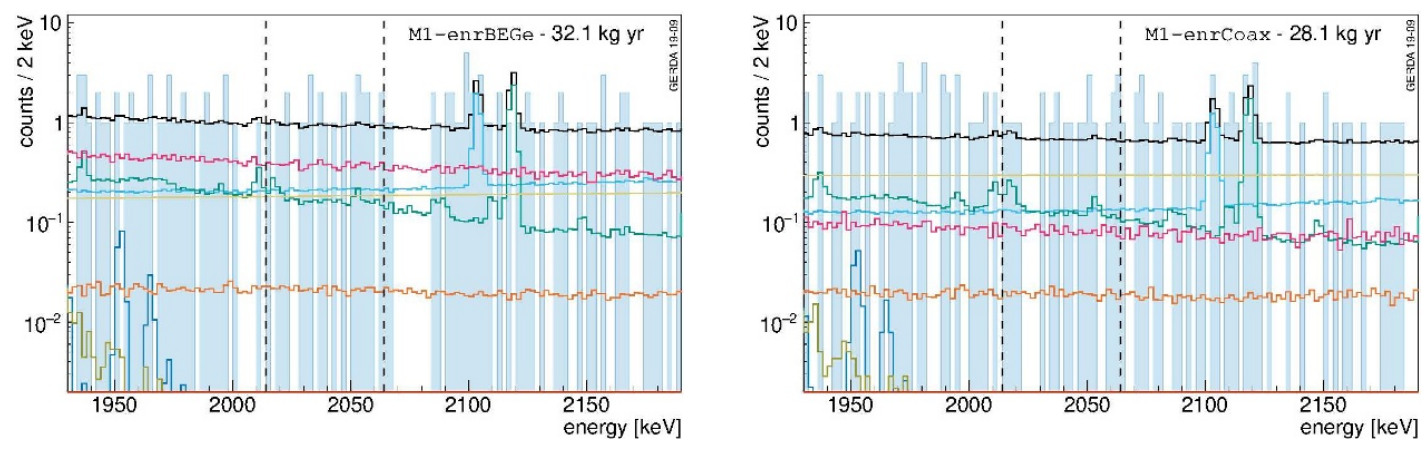

Figure 5. Background decomposition for the M1-enrBEGe (left) and the M1-enrCoax (right) data sets in the background window between $1930 \mathrm{keV}$ and $2190 \mathrm{keV}$ after data unblinding. The previously blinded window $\left(Q_{\beta \beta} \pm 25 \mathrm{keV}\right)$ is indicated by two dashed lines. The background distribution before active background suppression in the $0 \nu \beta \beta$ analysis window can be well approximated with a constant function. For color code see figure 4 .

\begin{tabular}{|cccc|}
\hline contamination & location 1 & location 2 & correlation \\
\hline${ }^{214} \mathrm{Bi}+{ }^{214} \mathrm{~Pb}$ & mini-shrouds & flat cables & -0.43 \\
& flat cables & detector holders & -0.45 \\
${ }^{40} \mathrm{~K}$ & flat cables & close to the array & -0.63 \\
& $\mathrm{LAr}-$ outside mini-shrouds & $\mathrm{n}^{+}$contact & -0.42 \\
${ }^{4} 2 \mathrm{~K}$ & $\mathrm{LAr}-$ outside mini-shrouds & LAr - above array & -0.56 \\
\hline
\end{tabular}

Table 3. Correlations between fit components relative to the same background contamination in different locations.

are $16.04_{-0.85}^{+0.78}$ (stat) $\cdot 10^{-3} \mathrm{cts} /(\mathrm{keV} \cdot \mathrm{kg} \cdot \mathrm{yr})$ for the $\mathrm{M} 1-\mathrm{enrBEGe}$ data set and $14.68_{-0.52}^{+0.47}$ (stat). $10^{-3} \mathrm{cts} /(\mathrm{keV} \cdot \mathrm{kg} \cdot \mathrm{yr})$ for the M1-enrCoax data set.

\section{Discussion}

In general, impurities close to the detector array contribute most to the background, far components give minor contributions. The posterior distribution and the screening measurements are in very good agreement and the spectral content of each source of background can be accounted for by the screened hardware components. Only in the case of ${ }^{40} \mathrm{~K}$ a large part of the observed activity cannot be explained by the screened hardware and is fit with the additionally introduced components far and close to the detector array. The ${ }^{42} \mathrm{~K}$ and $\alpha$ event distributions cannot be constrained by screening measurements and are adjusted to best fit the data.

The presented background model is not unambiguous in all components. As shown in figure 3 several PDFs of the same source of background located in different structural components are very similar and thus prone to correlation. Most of them have been resolved by introducing prior distributions based on the screening measurements. However, a few anti-correlations persist which are listed in table 3. 
For what concerns ${ }^{42} \mathrm{~K}$ in the LAr volume outside the mini-shrouds and thus more distant from the detector array, the adopted distribution is purely empirical. Our prior knowledge is limited by the fact that the ${ }^{42} \mathrm{~K}$ ions undergo drift due to the electrical fields surrounding the detectors and high-voltage cables. Also, due to thermal gradients they can be displaced by convection. Hence, their distribution inside the GERDA LAr is prone to systematic uncertainties. The presence of unshielded high-voltage cables above the detector array can explain the excess of ${ }^{42} \mathrm{~K}$ found in this region. From the perspective of the full-range fit a more sophisticated modeling does not significantly modify the ${ }^{42} \mathrm{~K}$ PDFs and hence the fit results. A potentially asymmetric ${ }^{42} \mathrm{~K}$ distribution is, thus, not further followed in the main analysis. Nevertheless, some considerations can be found in appendix A. An explanation for ${ }^{42} \mathrm{~K}$ on the $\mathrm{p}^{+}$contact being rejected for the $\mathrm{M} 1-\mathrm{enrBEGe}$ data set but potentially present in the M1-enrCoax data can be the specific bore-hole geometry of the semi-coaxial detectors. ${ }^{42} \mathrm{~K}$ produced inside the hole cannot easily escape and is trapped close to the $\mathrm{p}^{+}$contact.

For each source of background the contribution to the BI at $Q_{\beta \beta}$ prior to active background reduction is listed in table 2 . The statistical uncertainties on the single contributions to the BI are generally of the order of $10 \%$ or lower, with the exception of ${ }^{42} \mathrm{~K}$ and energydegraded $\alpha$ events, for which the uncertainty is roughly doubled. The two contributions are affected by a higher uncertainty because they are not bound by screening measurements.

The background event distribution in the $0 \nu \beta \beta$ analysis window can be well approximated with a constant function (see figure 5). With this assumption, the BIs extracted from data are $16.4_{-1.6}^{+1.7} \cdot 10^{-3} \mathrm{cts} /(\mathrm{keV} \cdot \mathrm{kg} \cdot \mathrm{yr})$ for M1-enrBEGe and $15.4_{-1.6}^{+1.8} \cdot 10^{-3} \mathrm{cts} /(\mathrm{keV} \cdot \mathrm{kg} \cdot \mathrm{yr})$ for the M1-enrCoax data set. These values agree well with the background model description presented in section 4 . The BIs prior to further analysis cuts and before the upgrade of the GERDA experiment to Phase II can be found in reference [41]. For the M1-enrCoax data set the BI prior to the upgrade of $(18 \pm 2) \cdot 10^{-3} \mathrm{cts} /(\mathrm{keV} \cdot \mathrm{kg} \cdot \mathrm{yr})$ is very consistent with the values presented here. The BI of the M1-enrBEGe data set instead is substantially improved from a Phase I value of $42_{-8}^{+10} \cdot 10^{-3} \mathrm{cts} /(\mathrm{keV} \cdot \mathrm{kg} \cdot \mathrm{yr})$ to a value which is at least $2.5 \times$ smaller in Phase II despite a significant increase of inactive hardware mass. ${ }^{5}$ Contributions to the BI from all isotopes have been improved with respect to Phase I with the exception of background introduced by $\alpha$ surface events. The most drastic improvement is notable for ${ }^{42} \mathrm{~K}$ for which the BI contribution for the ${ }^{\text {enr }}$ BEGe detectors appears four times smaller than before the upgrade to Phase II.

As mentioned in section 4 , the extracted $2 \nu \beta \beta$ half-life estimate is based on the M1-enrBEGe data set only. The additional parameter $\delta^{2 \nu}$ mainly quantifies the systematic biases between the active volume determination methods of the two detector types. The full charge collection depth (FCCD), which determines the active volume of a detector, was studied extensively in a detector characterization campaign for the ${ }^{\text {enr BEGe }}$ detectors $[12,13]$. The estimate of the FCCD used in this analysis is based on measurements using an ${ }^{241} \mathrm{Am}$ source with characteristic $\gamma$ lines at $60 \mathrm{keV}, 99 \mathrm{keV}$ and $103 \mathrm{keV}$.

\footnotetext{
${ }^{5}$ Note the slight difference of the M1-enrBEGe analysis data set presented here and the data set used for $0 \nu \beta \beta$ analysis for which the improvement in the BI is slightly higher $(3 \times$ better $\mathrm{BI})$. This is due to discarded ${ }^{\text {enr }}$ BEGe data for which no PSD can be applied.
} 
However, the FCCD was also measured using a ${ }^{60}$ Co source with characteristic $\gamma$ energies of $1173 \mathrm{keV}$ and $1332 \mathrm{keV}$. The latter FCCD $\mathrm{Co}_{\mathrm{o}}$ is systematically higher (about $3 \%$ ) with respect to the $\mathrm{FCCD}_{\mathrm{Am}}$. The discrepancy could be explained by an energy dependence of the initial charge-carrier cloud size inside the detector but the actual impact on the active volume is still under investigation. For the ${ }^{\text {enr }}$ Coax detectors only FCCD values determined with a ${ }^{60} \mathrm{Co}$ source are available. Considering the systematic uncertainties affecting the determined active ${ }^{76} \mathrm{Ge}$ exposures of the M1-enrBEGe and M1-enrCoax data sets (1.8\% and $5 \%$ respectively, see table 1) $\delta^{2 \nu}$ is compatible with zero within $1 \sigma .{ }^{6}$

Various systematic effects have to be considered when estimating the uncertainty on the $2 \nu \beta \beta$ half-life $T_{1 / 2}^{2 \nu}$. Due to the fact that the aim of the paper is not a precise $2 \nu \beta \beta$ half-life measurement, for most of them only a conservative evaluation is provided. Several systematic uncertainties arise from the Monte Carlo simulation framework. Uncertainties due to the GEANT4 model of particle interactions and propagation were estimated to be of the order of $2 \%$ in previous publications [42, 43]. Approximations in the implementation of the GERDA setup are conservatively estimated within a 1-2\% uncertainty range. This accounts for possible spectral shape modifications due to inaccurate charge collection model between the $\mathrm{n}^{+}$contact layer and the active detector volume. Uncertainties induced by the theoretical model of $2 \nu \beta \beta$ decays implemented in DECAY0, as well as data acquisition and selection methods are considered negligible. A $1.8 \%$ contribution accounts for uncertainties in the enrichment and active mass fraction determination (see active ${ }^{76} \mathrm{Ge}$ exposure in table 1). All the systematic effects considered above sum up to a total systematic uncertainty on $T_{1 / 2}^{2 \nu}$ of $3-4 \%$. In total this leads to $T_{1 / 2}^{2 \nu}=(2.03 \pm 0.09) \cdot 10^{21} \mathrm{yr}$ compatible with earlier results [42, 43].

\section{Conclusions}

We presented the background decomposition of GERDA Phase II data before the application of active background suppression techniques using a multivariate Bayesian fit approach based on single- and two-detector data in energy and detector space. The model is able to well describe the data and the results are compatible with the expectations from material screening measurements. The only exception is ${ }^{40} \mathrm{~K}$ for which a higher contamination is found, dominantly in hardware components close to the detector array. This indicates contaminations introduced during production and mounting procedures different from the screened reference samples; in fact a few parts underwent further processing after material screening. Analyzing the count rates in the ${ }^{40} \mathrm{~K}$ and ${ }^{42} \mathrm{~K}$ high-statistics $\gamma$ lines on a detectorby-detector basis we find indications for asymmetries in the spatial distribution of the two potassium isotopes. Furthermore, the background indices at $Q_{\beta \beta}$ prior active background suppression techniques are given by

$$
\begin{aligned}
{ }^{\text {enr }} \text { BEGe } & 16.04_{-0.85}^{+0.78}(\mathrm{stat}) \cdot 10^{-3} \mathrm{cts} /(\mathrm{keV} \cdot \mathrm{kg} \cdot \mathrm{yr}) \\
{ }_{\text {enr }} \text { Coax } & 14.68_{-0.52}^{+0.47}(\mathrm{stat}) \cdot 10^{-3} \mathrm{cts} /(\mathrm{keV} \cdot \mathrm{kg} \cdot \mathrm{yr})
\end{aligned}
$$

\footnotetext{
${ }^{6}$ The systematic bias between the active volume estimates for the BEGe and coaxial detector types is a sub-dominant contribution in the $0 \nu \beta \beta$ analysis with respect to e.g. PSD uncertainties.
} 
and are in very good agreement with the assumption of a flat background distribution in this region. In terms of the BI the upgrade to GERDA Phase II proves extremely successful. Despite major hardware changes and higher inactive mass close to the detectors, the BI before applying active background reduction remains unchanged for the ${ }^{\text {enr }}$ Coax detectors and is improved by a factor of three for the ${ }^{\text {enr }}$ BEGe detectors.

A careful background model is essential in order to separate the two-neutrino doublebeta decay events from the other background components. We expect to substantially improve the precision of the $T_{1 / 2}^{2 \nu}$ measurement after applying the LAr veto cut. In this manner, the signal to background ratio in the $2 \nu \beta \beta$ energy region is improved by about an order of magnitude [7,8]. Furthermore, this allows precision studies of the shape of the $2 \nu \beta \beta$ spectrum and hence to test physics models beyond the Standard Model such as $0 \nu \beta \beta$ decay with Majoron emission and Lorentz symmetry violation effects [43, 44].

The localization of impurities makes the exchange of particularly contaminated components possible in upgrade works and thus the background can be potentially lowered even further. Moreover, it is important to learn what are the most important sources of background in order to improve handling and cleaning procedures as well as material selection. For future experiments like the Large Enriched Germanium Experiment for Neutrinoless $\beta \beta$ Decay (LEGEND) [45], which aims to cover the parameter space of inverted neutrino mass hierarchy, background reduction is the most crucial step in achieving the necessary sensitivity. The goal is to achieve a background index one order of magnitude lower than GERDA Phase II.

\section{Acknowledgments}

The Gerda experiment is supported financially by the German Federal Ministry for Education and Research (BMBF), the German Research Foundation (DFG) via the Excellence Cluster Universe and the SFB1258, the Italian Istituto Nazionale di Fisica Nucleare (INFN), the Max Planck Society (MPG), the Polish National Science Centre (NCN), the Foundation for Polish Science (TEAM/2016-2/2017), the Russian Foundation for Basic Research (RFBR), and the Swiss National Science Foundation (SNF). The institutions acknowledge also internal financial support. This project has received funding or support from the European Union's Horizon 2020 research and innovation programme under the Marie Sklodowska-Curie Grant Agreements No. 690575 and No. 674896, respectively. The GERDA collaboration thanks the directors and the staff of the LNGS for their continuous strong support of the GERDA experiment.

\section{A Potassium tracking analysis}

The two full-energy lines of ${ }^{40} \mathrm{~K}$ and ${ }^{42} \mathrm{~K}$ at $1461 \mathrm{keV}$ and $1525 \mathrm{keV}$ are distinct features of the energy spectrum shown in figure 2. Being a relevant source of background for doublebeta decay, the two potassium isotopes play a crucial role in the background modeling process in GERDA. Uncertainties in their origin and distribution propagate directly to searches for exotic physics like Majorons, Lorentz invariance-violating processes or decay 
modes to excited states of $2 \nu \beta \beta$ decay in which the shape of the $2 \nu \beta \beta$ decay spectrum is a unique feature and thus need to be well understood.

Initial observations in Phase II have shown that the ${ }^{40} \mathrm{~K}$ and ${ }^{42} \mathrm{~K}$ full-energy line intensities have increased by a factor of 4 and 2, respectively, in the single-detector data compared to Phase I [46]. The ${ }^{42} \mathrm{~K}$ increase in activity can be attributed to the exchange of the mini-shrouds material from copper to nylon ${ }^{7}$ during the Phase II upgrade: the electric field generated by the detectors bias high voltage is not screened by the conductive material anymore. The ${ }^{42} \mathrm{~K}$ ions can be attracted from a larger LAr volume into the vicinity of the detectors. Moreover, the unshielded high-voltage cables could be an explanation for the higher rate of ${ }^{42} \mathrm{~K}$ events seen in the uppermost detectors in the GERDA array. The higher ${ }^{40} \mathrm{~K}$ event rate, on the other hand, is possibly attributable to the glue used for the nylon mini-shrouds and other new materials introduced with the LAr veto system. The exact amount, location and radio-purity of the glue is not precisely known. All changes to the setup that have been made during the upgrade to Phase II are described and motivated in exhaustive detail in reference [5].

In the following sections we focus on the characteristics of the events constituting the two potassium lines. In order to extract information about the spatial distribution of ${ }^{40} \mathrm{~K}$ and ${ }^{42} \mathrm{~K}$ contamination around the GERDA array, a treatment on a detector-by-detector basis is advantageous. The two $\gamma$ lines contain enough statistics for such an analysis to be meaningful and constitute samples with a high signal to background ratio.

\section{A.1 Data}

Two windows around the potassium $\gamma$ lines are projected in detector index space, such that, for single-detector data, each data point $n_{i}$ represents the total counts in detector $i$ in the respective energy window. For two-detector data the detector space is two-dimensional, and each data point $n_{i j}$ represents the number of events for which energy is deposited in detector $i$ and detector $j$.

The events in the potassium lines (denoted with $\mathrm{K} 40$ and $\mathrm{K} 42$ in the following) are selected in a $\pm 3 \sigma$ energy interval around the respective line, rounded up to an integer number of $\mathrm{keV}$ to match the specific energy windows in the energy distributions with $1 \mathrm{keV}$ binning. $\sigma$ is the energy resolution in the respective energy window. Additionally, three side-bands (SB1, SB2 and SB3 in the following) are used to estimate the continuum below and above the $\gamma$ lines. Considering the further subdivision in single- (M1-) and two-detector (M2-) data, this leads to the definition of $5 \times 2$ energy regions, summarized in table 4 . A visual representation of the selected windows can be found in figure 6 . We use the PDFs respective to ${ }^{214} \mathrm{Bi}$ on the flat cables and detector intrinsic $2 \nu \beta \beta$ decays to estimate the background. Other components are expected to contribute less in the respective energy windows.

\footnotetext{
${ }^{7}$ The exchange of material from copper to transparent nylon was necessary in order to properly propagate the LAr scintillation light from inside the mini-shrouds to the light detectors.
} 


\begin{tabular}{|ccccc|}
\hline & M1- $[\mathrm{keV}]$ & cts. & M2- $[\mathrm{keV}]$ & cts. \\
\hline K40 & {$[1457,1465]$} & 4472 & {$[1455,1467]$} & 554 \\
K42 & {$[1521,1529]$} & 6718 & {$[1519,1531]$} & 865 \\
\hline SB1 & {$[1405,1450]$} & 1852 & {$[1405,1450]$} & 452 \\
SB2 & {$[1470,1515]$} & 1124 & {$[1470,1515]$} & 326 \\
SB3 & {$[1535,1580]$} & 533 & {$[1535,1580]$} & 41 \\
\hline
\end{tabular}

Table 4. Energy ranges and corresponding number of events for the potassium tracking analysis (visualized in figure 6). Note that the windows for two-detector data are larger as the two singledetector energy resolutions are folded in the summed energy spectrum.

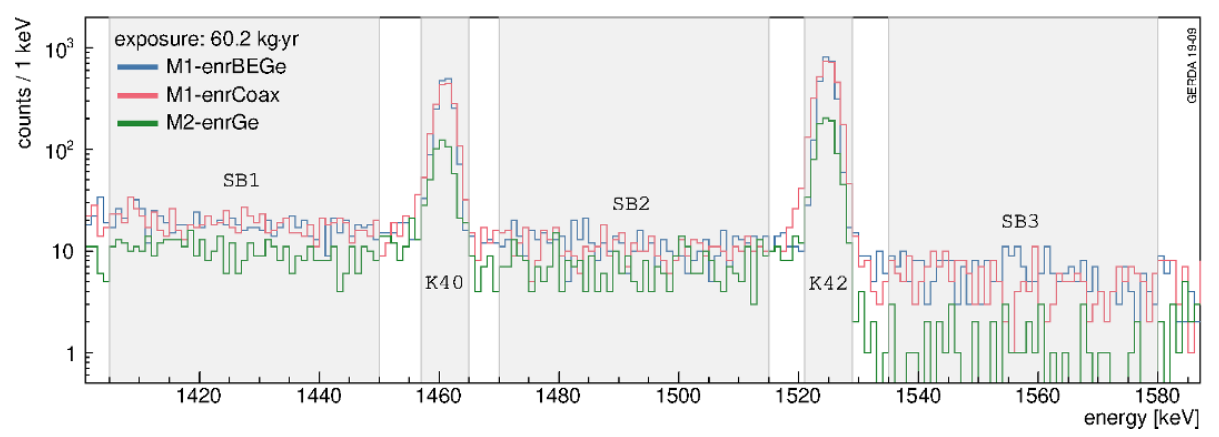

Figure 6. Visual representation of the five energy ranges defined for the potassium tracking analysis. The exact intervals and counts are given in table 4 .

\section{A.2 Analysis}

The statistical approach of factorizing the likelihood is described in section 3.2. The part of the likelihood we are analyzing here runs simultaneously on the $5 \times 2$ energy ranges presented above. Following the naming convention introduced in section 3 it reads:

$$
\mathcal{L}_{\mathrm{K}}\left(\lambda_{1}, \ldots, \lambda_{m^{\prime}} \mid n\right)=\prod_{d=1}^{N_{\mathrm{dat}}}\left\{\prod_{i=1}^{N_{\mathrm{det}}} \operatorname{Pois}\left(n_{d, i}^{\mathrm{M} 1} ; \nu_{d, i}^{\mathrm{M} 1}\right) \times \prod_{j<k}^{N_{\mathrm{det}}} \operatorname{Pois}\left(n_{d, j k}^{\mathrm{M} 2} ; \nu_{d, j k}^{\mathrm{M} 2}\right)\right\}
$$

where the index $i$ runs over the bins (i.e. detectors) and the index $d$ over the 5 energy windows considered, namely the three side-bands SB1, SB2, SB3 and the two line-bands K40 and K42. The M2- data sets are two-dimensional in detector space and run over the two indices $j$ and $k$.

Gaussian prior probability distributions for the ${ }^{40} \mathrm{~K}$ activity are built from radio-purity screening measurements (see reference [5] section 5). For ${ }^{42} \mathrm{~K}$, for which no screening information is available, uniform priors are adopted, with the exception of the two ${ }^{42} \mathrm{~K}$ components located on the $\mathrm{n}^{+}$contact surface of enr BEGe and ${ }^{\text {enr }}$ Coax detectors. ${ }^{42} \mathrm{~K}$ can be attracted to the $\mathrm{n}^{+}$surface by the electrical field created by the high voltage potential applied to the detectors. Both components are expected to be correlated by the volume ratio of the mini-shrouds (3:2 ${ }^{\text {enr }} \mathrm{BEGe}$ to $\left.{ }^{\mathrm{enr}} \mathrm{Coax}\right)$ the ${ }^{42} \mathrm{~K}$ ions are attracted from. The volume ratio estimate is extracted from the geometric implementation in MAGE. We 
assume an uncertainty of $0.1 \mathrm{mBq}$ on either activity allowing for a change of their ratio. The correlation is included in the fit via a two-dimensional prior.

The analysis flow starts with a construction of a first, preliminary model, which consists only of background contributions that are expected from screening measurements of ${ }^{40} \mathrm{~K}$ and known properties of ${ }^{42} \mathrm{~K}$. The resulting model, however, gives a non-satisfactory description of data and the posterior distributions for the ${ }^{40} \mathrm{~K}$ components are significantly shifted to higher values with respect to the prior distributions, indicating a surplus of ${ }^{40} \mathrm{~K}$.

To find a better agreement with physics data while keeping the model as simple as possible, additional components using uniform priors are included one at a time in the fitting procedure, and the Bayes factor is calculated between the extended and the preliminary model. The model is iteratively updated by adding the component that results in the highest Bayes factor until no Bayes factor is larger than 10.

In a first iteration a replica of the PDF of ${ }^{40} \mathrm{~K}$ in the mini-shrouds is added obtaining a Bayes factor $\gg 10 .{ }^{40} \mathrm{~K}$ in the Tetratex ${ }^{\circledR}$-coated copper shrouds is added in a second iteration with a Bayes factor of 11 . For ${ }^{42} \mathrm{~K}$ the only additional component that results in a Bayes factor greater than 1 is ${ }^{42} \mathrm{~K}$ on the $\mathrm{n}^{+}$detector contacts. Although the fit shows only a slight preference (Bayes factor of 2) the component is added to the model because of its importance in the full-range fit, where the energy region above the $1525 \mathrm{keV} \gamma$ line is also considered.

The results of the base model are shown in table 5 and a graphic representation showing the counts per detector in both potassium $\gamma$ lines in M1- and M2-data can be found in figure 7 . The analysis yields a $p$-value of $\sim 0.07$, indicating an acceptable description of the data. To further improve the model rotationally asymmetric fit components are needed. The base model is accurate enough to be used as input for the full-range fit, which is insensitive to any rotational inhomogeneity of the location of background sources, as spectra from different detectors are merged into a single data set.

The two components ${ }^{40} K$ close to the array and ${ }^{42} K$ in $L A r$ - above the array are split into 7 sub-components on a string-by-string basis (for the respective PDFs see appendix C). Furthermore, we consider a ${ }^{40} \mathrm{~K}$ contamination on top of the central mini-shroud.

The results of this extended analysis are listed in table 6 . An elevated ${ }^{42} \mathrm{~K}$ concentration is found above the central string while a lower concentration is observed above the adjacent strings S1 and S6 (string numbers follow the nomenclature used in figure 8). Due to the large number of components the fit yields a high anti-correlation between the ${ }^{42} \mathrm{~K}$ concentration above the outer strings and S7. This results in a high uncertainty on the latter fit parameter.

The screening measurements do not account for all observed ${ }^{40} \mathrm{~K}$. In general ICP-MS screening of the mini-shrouds with respect to ${ }^{40} \mathrm{~K}$ is difficult and yielded only a lower limit. Different measurements seem to indicate different contamination levels of different mini-shrouds. Samples of glued nylon yielded the highest potassium contamination. As the gluing of the nylon mini-shrouds is done manually during installation the amount of glue and its exact location is hard to control. Hence, an asymmetric distribution is expected. The ${ }^{40} \mathrm{~K}$ content of other close components like holders and cables might also be asymmetric. The asymmetric ${ }^{40} \mathrm{~K}$ contamination is confirmed by the extended potassium 

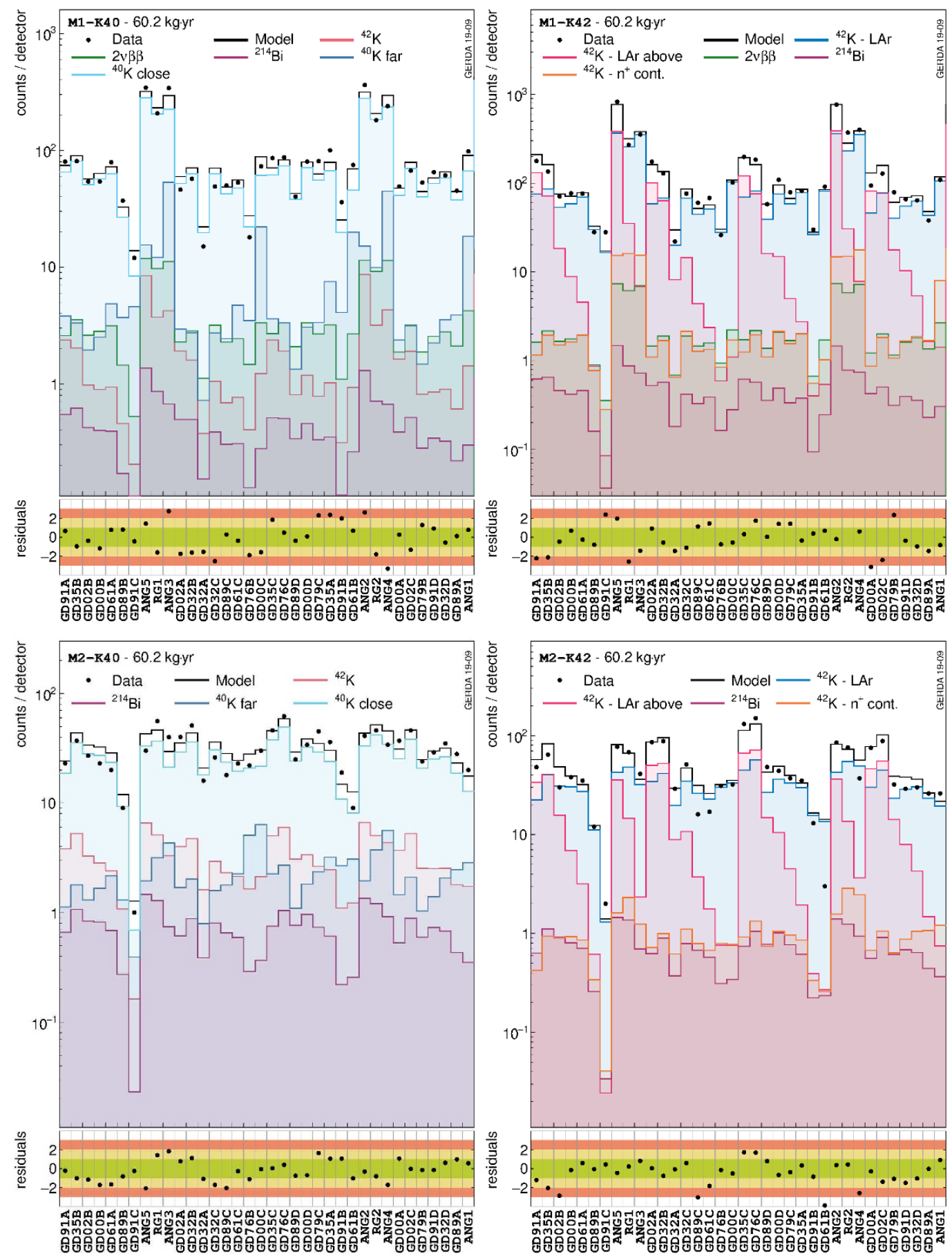

Figure 7. Decomposition of the energy windows corresponding to the two potassium lines in detector space: single-detector data (top) one-dimensional representation of two-detector data (bottom). Some components are merged for visualization purposes: in the K40 plots combined components are shown for ${ }^{42} \mathrm{~K}$ and ${ }^{214} \mathrm{Bi}$, while ${ }^{40} \mathrm{~K}$ sources are grouped in close (flat cables, holders, mini-shrouds) and far (fibers, SiPMs, copper shrouds, front-end electronics) locations from the detector array. To visualize the two-detector data the sum of the projections on the two domain axes (index $i$ and index $j$ ) is shown. 


\begin{tabular}{|c|c|c|c|c|c|}
\hline source & [prior] location & units & $\begin{array}{l}\text { global } \\
\text { mode }\end{array}$ & $\begin{array}{l}\text { marg. } \\
\text { mode }\end{array}$ & $\begin{array}{c}68 \% \text { C.I. or } \\
90 \% \text { upper C.L. }\end{array}$ \\
\hline \multirow{9}{*}{${ }^{40} \mathrm{~K}$} & [g] flat cables & \multirow{9}{*}{$\mathrm{mBq}$} & 3.29 & 3.25 & {$[1.79,4.72]$} \\
\hline & [g] front-end electronics & & 15.7 & 15.9 & {$[11.1,20.1]$} \\
\hline & [g] copper shrouds ${ }^{\dagger}$ & & 18.4 & 18.1 & {$[16.6,20.0]$} \\
\hline & [g] fiber shroud & & 2.82 & 2.81 & {$[2.24,3.38]$} \\
\hline & [g] detector holders & & 1.73 & 1.73 & {$[1.28,2.14]$} \\
\hline & [g] mini-shrouds & & 1.70 & 1.70 & {$[1.60,1.80]$} \\
\hline & [g] SiPM ring & & 2.50 & 2.73 & {$[0.83,4.13]$} \\
\hline & [f] far from the array & & 328 & 322 & {$[232,416]$} \\
\hline & [f] close to the array & & 10.8 & 10.8 & {$[9.53,12.1]$} \\
\hline \multirow{4}{*}{${ }^{42} \mathrm{~K}$} & {$[\mathrm{f}] \mathrm{n}^{+}(\mathrm{BEGe})$} & \multirow{4}{*}{$\mathrm{mBq}$} & 0 & 0 & $<0.37$ \\
\hline & [f] $\mathrm{n}^{+}(\operatorname{Coax})$ & & 0.22 & 0.24 & {$[0.12,0.38]$} \\
\hline & [f] LAr - above array & & 450 & 454 & {$[436,470]$} \\
\hline & [f] LAr - outside mini-shrouds & & 2036 & 2009 & {$[1915,2080]$} \\
\hline${ }^{214} \mathrm{Bi}$ & [g] flat cables & $\mathrm{mBq}$ & 1.51 & 1.26 & {$[0.93,1.51]$} \\
\hline $2 \nu \beta \beta$ & [f] germanium & $10^{21} \mathrm{yr}$ & 1.91 & 1.93 & {$[1.86,2.00]$} \\
\hline
\end{tabular}

$\dagger$ Tetratex ${ }^{\circledR}$-coated.

Table 5. Summary of the fit parameters estimated with the potassium source tracking analysis (base model). The type of prior distribution is indicated with $[\mathrm{f}]$ : flat, $[\mathrm{g}]$ : Gaussian.

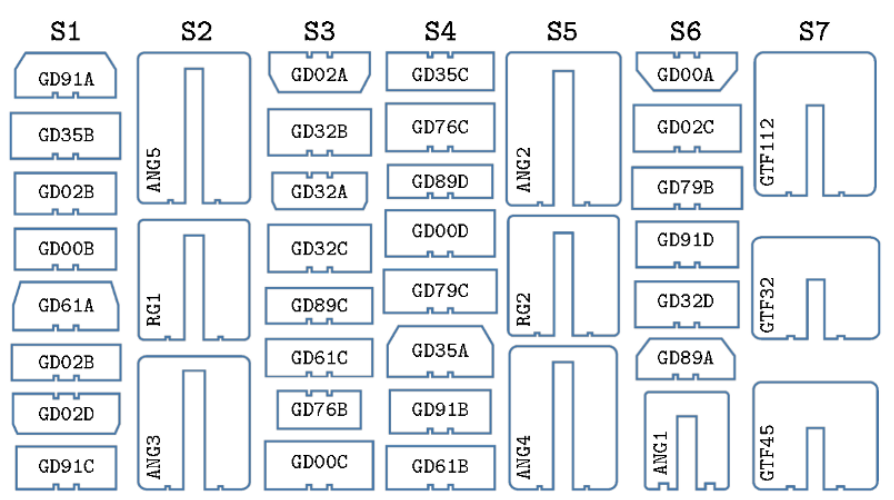

Figure 8. Detector string configuration in the GERDA array. Names prefixed with GD refer to detectors of ${ }^{\text {enr } B E G e}$ type whereas ANG and RG refer to ${ }^{\text {enr }}$ Coax detectors. The three natural coaxial detectors (prefixed with GTF) which are located in the central string S7 are not used in this analysis. 


\begin{tabular}{|c|c|c|c|c|c|}
\hline source & [prior] location & units & $\begin{array}{l}\text { global } \\
\text { mode }\end{array}$ & $\begin{array}{l}\text { marg. } \\
\text { mode }\end{array}$ & $\begin{array}{c}68 \% \text { C.I. or } \\
90 \% \text { upper C.L }\end{array}$ \\
\hline \multirow{16}{*}{${ }^{40} \mathrm{~K}$} & [g] flat cables & \multirow{16}{*}{$\mathrm{mBq}$} & 2.33 & 1.08 & {$[0.13,2.30]$} \\
\hline & [g] front-end electronics & & 14.5 & 14.4 & {$[10.2,18.7]$} \\
\hline & [g] copper shrouds ${ }^{\dagger}$ & & 18.4 & 18.5 & {$[16.6,20.0]$} \\
\hline & [g] fiber shroud & & 2.83 & 2.77 & {$[2.24,3.38]$} \\
\hline & [g] detector holders & & 2.57 & 2.29 & {$[1.75,2.78]$} \\
\hline & [g] mini-shrouds & & 1.70 & 1.70 & {$[1.60,1.79]$} \\
\hline & [f] close to $\mathrm{S} 1$ & & 0.81 & 0.83 & {$[0.47,1.28]$} \\
\hline & [f] close to S2 & & 2.35 & 2.22 & {$[1.83,2.51]$} \\
\hline & [f] close to S3 & & 0 & 0 & $<0.50$ \\
\hline & [f] close to $\mathrm{S} 4$ & & 2.58 & 2.55 & {$[2.10,3.02]$} \\
\hline & [f] close to S5 & & 0.97 & 0.85 & {$[0.56,1.16]$} \\
\hline & [f] close to $\mathrm{S} 6$ & & 1.86 & 1.89 & {$[1.46,2.30]$} \\
\hline & [f] close to $\mathrm{S} 7$ & & 0 & 0 & $<2.92$ \\
\hline & [f] S7 mini-shroud (top) & & 2.09 & 1.83 & {$[1.26,2.40]$} \\
\hline & [g] SiPM ring & & 2.44 & 2.32 & {$[0.83,4.02]$} \\
\hline & [f] far from the array & & 390 & 374 & {$[280,468]$} \\
\hline \multirow{10}{*}{${ }^{42} \mathrm{~K}$} & [f] $\mathrm{n}^{+}(\mathrm{BEGe})$ & \multirow{10}{*}{$\mathrm{mBq}$} & 0.15 & 0.19 & {$[0.05,0.37]$} \\
\hline & {$[\mathrm{f}] \mathrm{n}^{+}(\operatorname{Coax})$} & & 0.22 & 0.26 & {$[0.12,0.41]$} \\
\hline & [f] LAr - above S1 & & 0 & 0 & $<0.80$ \\
\hline & [f] LAr - above S2 & & 2.22 & 2.96 & {$[2.21,3.63]$} \\
\hline & [f] LAr - above S3 & & 1.20 & 1.57 & {$[1.06,2.16]$} \\
\hline & [f] LAr - above S4 & & 1.43 & 1.89 & {$[1.33,2.41]$} \\
\hline & [f] LAr - above S5 & & 1.49 & 1.91 & {$[1.38,2.73]$} \\
\hline & [f] LAr - above S6 & & 0 & 0 & $<1.21$ \\
\hline & [f] LAr - above S7 & & 10.4 & 7.84 & {$[4.95,9.83]$} \\
\hline & [f] LAr - outside mini-shrouds & & 2083 & 2058 & {$[1960,2145]$} \\
\hline${ }^{214} \mathrm{Bi}$ & [g] flat cables & $\mathrm{mBq}$ & 1.60 & 1.41 & {$[1.14,1.66]$} \\
\hline $2 \nu \beta \beta$ & [f] germanium & $10^{21} \mathrm{yr}$ & 1.89 & 1.89 & {$[1.83,1.97]$} \\
\hline
\end{tabular}

\footnotetext{
$\dagger$ Tetratex $^{\circledR}$-coated.
}

Table 6. Summary of the fit parameters estimated with the potassium source tracking analysis (extended model). The type of prior distribution is indicated with $[\mathrm{f}]$ : flat, $[\mathrm{g}]$ : Gaussian. 
tracking analysis. Also, an additional ${ }^{40} \mathrm{~K}$ distribution on the top-lid of the central minishroud is preferred. The surplus far ${ }^{40} \mathrm{~K}$ component instead is possibly explained by setup parts omitted in the model like the PMTs and voltage-dividers of the LAr veto system. An upper limit of their ${ }^{40} \mathrm{~K}$ content, $<330 \mathrm{mBq}$, was estimated from material screening which is similar to the activity reconstructed for the far ${ }^{40} \mathrm{~K}$ component. The location of the PMTs with respect to the detector array is very similar to the Tetratex ${ }^{\circledR}{ }_{\text {-coated }}$ copper-shrouds and their PDFs are, hence, degenerate.

\section{B $\quad \alpha$ events background analysis}

Above an energy of $3.5 \mathrm{MeV}$ almost all registered events are due to $\alpha$ emitting isotopes. The respective part of the full likelihood can be approximately factorized and studied separately. $\alpha$ particles have a very short range in LAr as well as in germanium (continuous slowing down approximation, CSDA, range of $50 \mu \mathrm{m}$ and $20 \mu \mathrm{m}$, respectively [47]) and are able to reach a detector's active volume only through the very thin (of the order of $500 \mathrm{~nm}) \mathrm{p}^{+}$contact surface. Therefore, the $\alpha$ emitter contamination is detector-specific and depends only on the $\mathrm{p}^{+}$surface contaminations. Therefore, we analyze the ${ }^{\text {enr }}$ BEGe

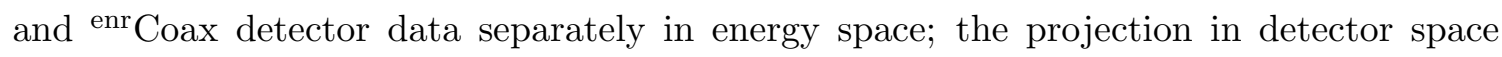
bares no correlation between detectors and hence contains no further useful information. The number of events in a single detector is not sufficient to further split the data on a detector-by-detector basis. The two data sets are uncorrelated and the statistical analysis can be carried out for each single-detector data set separately. In the two-detector data the $\alpha$ component is not observed due to the short range of these particles.

All contaminations found are constituents of the ${ }^{238} \mathrm{U}$ decay chain. The main surface contamination observed is ${ }^{210} \mathrm{Po}$ which occurs either as an incident contamination and decays in time with a half-life of $138.3763(17)$ days [48] or is fed by a contamination with ${ }^{210} \mathrm{~Pb}$ with a stable rate in time. The spectral form is identical for both cases and can only be disentangled by analyzing the $\alpha$ rate in time (see section B.1).

Above the ${ }^{210}$ Po peak very few events are observed. In the M1-enrBEGe data set we find only four events with an energy larger than $5.3 \mathrm{MeV}$, while in the M1-enrCoax data set 22 such events are observed, 14 of which in a single detector ANG2 (see table 7). These events are due to $\alpha$ decays from ${ }^{222} \mathrm{Rn}$ and subsequent isotopes on the $\mathrm{p}^{+}$detector surfaces. ANG2 also shows a higher ${ }^{226} \mathrm{Ra}$ (mother nucleus of ${ }^{222} \mathrm{Rn}$ ) contamination which suggests dominantly a surface contamination with ${ }^{226} \mathrm{Ra}$ rather than ${ }^{222} \mathrm{Rn}$ dissolved in the LAr. In the latter case the decay chain would be broken as only the gaseous ${ }^{222} \mathrm{Rn}$ emanates from the construction materials into the LAr. The number of counts is too low to distinguish the spectral shape above $5.3 \mathrm{MeV}$ and disentangle a surface contamination with ${ }^{226} \mathrm{Ra}$ from ${ }^{222} \mathrm{Rn}$ dissolved in LAr. A comparison between the counts observed above $5.3 \mathrm{MeV}$ and the ${ }^{214} \mathrm{Bi} 609 \mathrm{keV} \gamma$ line suggests that $\alpha$ events due to a dissolved ${ }^{222} \mathrm{Rn}$ contamination would not produce observable counts in said energy region. Assuming that all ${ }^{214} \mathrm{Bi}$ observed comes from dissolved ${ }^{222} \mathrm{Rn}$ leads, in fact, to a specific activity smaller than $10 \mu \mathrm{Bq} / \mathrm{kg}$. Hence, in the following, we will only consider a $\mathrm{p}^{+}$surface contamination with ${ }^{226} \mathrm{Ra}$ and 


\begin{tabular}{|cccc|}
\hline data set & detector & channel & ${ }^{226}$ Ra-chain [cts] \\
\hline \multirow{5}{*}{ M1-enrBEGe } & GD61C & 16 & 1 \\
& GD79B & 32 & 1 \\
& GD89A & 35 & 2 \\
\hline \multirow{5}{*}{ M1-enrCoax } & ANG1 & 36 & 2 \\
& ANG2 & 27 & 14 \\
& ANG3 & 10 & 1 \\
& ANG4 & 29 & 1 \\
& ANG5 & 8 & 2 \\
& RG1 & 9 & 2 \\
\hline
\end{tabular}

Table 7. Observed number of counts with energy $>5.3 \mathrm{MeV}$ belonging to the ${ }^{226}$ Ra decay chain. Detectors with zero counts are not listed.

all subsequent isotopes to which we refer as the ${ }^{226} \mathrm{Ra}$ decay chain. The ${ }^{210} \mathrm{Po}$ and ${ }^{226} \mathrm{Ra}$ contaminations are not necessarily spatially correlated.

Due to the very short range of $\alpha$ particles the energy spectrum of $\alpha$ decays exhibits a line with a pronounced low-energy tail. The tail is formed when the decay occurs under an incident angle with respect to the contact and the $\alpha$ particle loses part of its energy before reaching the detectors active volume. The maximum is shifted with respect to the full emission energy which is due to energy loss inside the electrode and depends on its minimal thickness. The detectors have slightly different contact thicknesses, also, the $\mathrm{p}^{+}$ contact of a single detector may intrinsically be inhomogeneous. Therefore, we model the ${ }^{210}$ Po peak with a mixture of PDFs obtained from simulations with different contact thicknesses. Due to the low number of counts observed in the ${ }^{226} \mathrm{Ra}$ chain it is sufficient to model this component with only one PDF. Furthermore, the isotope contamination is assumed to halve at each decay step. A reduction effect of the subsequent $\alpha$ decays in the ${ }^{222} \mathrm{Rn}$ chain had been observed in Phase I and attributed to possible recoil off the surface into the LAr [14]. We adopt this explanation in our model although we note that the number of events observed with an energy $>5.3 \mathrm{MeV}$ is not sufficient to confirm the previously observed reduction effect. Further details about the construction of the PDFs are given in appendix $\mathrm{C}$.

Dedicated measurements [49] have shown that events originating in the contact separating groove are partly reconstructed with degraded energy. A simulation-based model of these energy-degraded events is not available yet. We approximate this component with an empirical linear distribution truncated below the maximum of the ${ }^{210}$ Po peak. Such a component accommodates also eventual $\alpha$ decays in the LAr in very close vicinity to the $\mathrm{p}^{+}$detector surface. However, the number of events found with an energy $>5.3 \mathrm{MeV}$ is too low to fully account for the linearly modeled distribution.

The likelihood function for modeling the high-energy region dominated by $\alpha$ decays runs only on single-detector data, namely M1-enrBEGe and M1-enrCoax separately, in a 

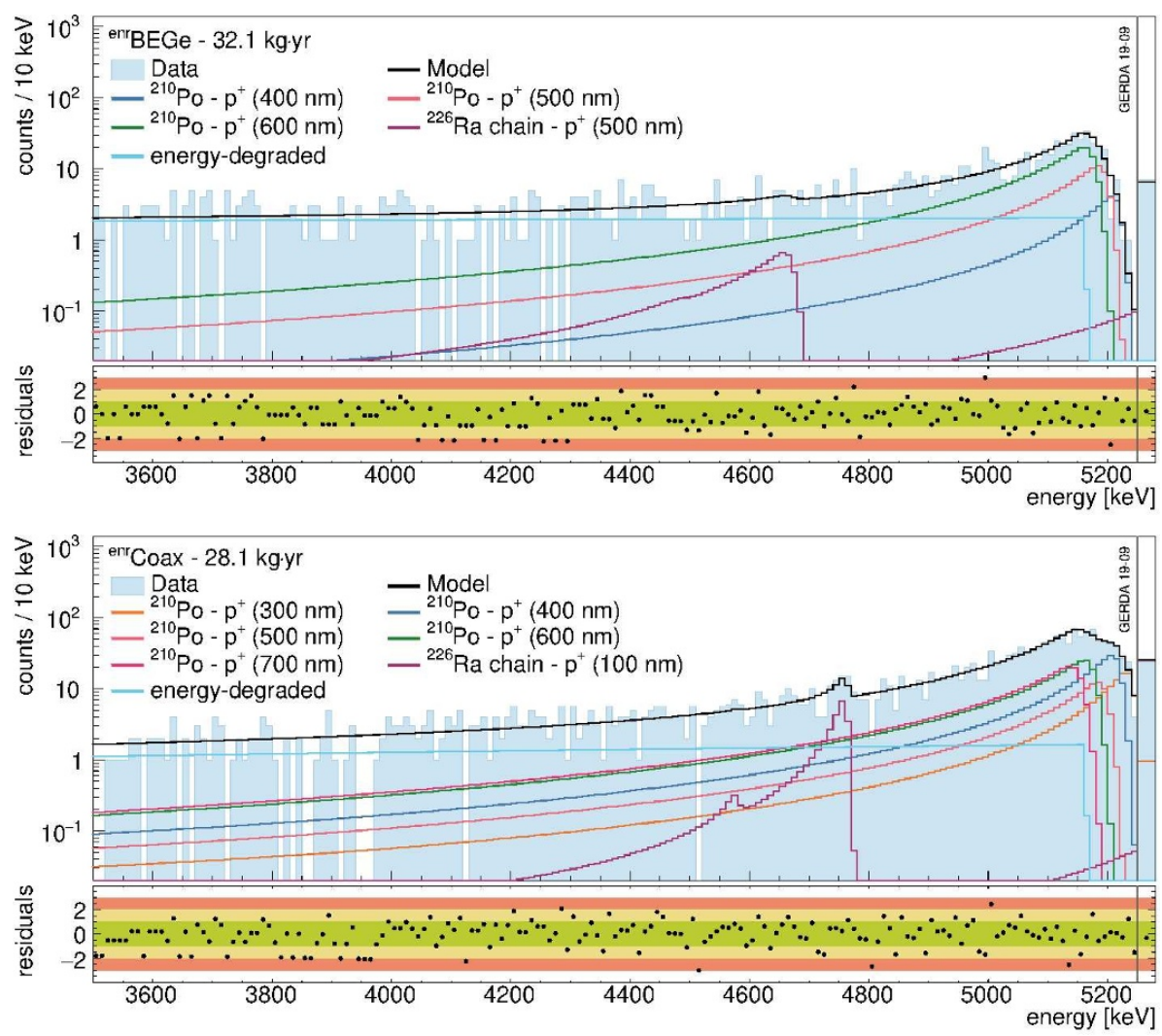

Figure 9. Fit results of the $\alpha$ events background analysis for M1-enrBEGe (top) and M1-enrCoax (bottom). The last bin contains all events above $5250 \mathrm{keV}$.

range from $3.5 \mathrm{MeV}$ to $5.25 \mathrm{MeV}$. Events with an energy higher than $5.25 \mathrm{MeV}$ are put in a single overflow bin:

$$
\mathcal{L}_{\alpha}\left(\lambda_{1}, \ldots, \lambda_{m} \mid n\right)=\prod_{i=1}^{N_{\text {bins }}} \operatorname{Pois}\left(n_{i} ; \nu_{i}\right)
$$

A flat prior probability is assigned to each of the fit parameters $\lambda_{i}$. Both data sets are fit separately with a fixed bin size of $10 \mathrm{keV}^{8}$ as the $\alpha$ contamination is detector individual and the two single-detector data sets are uncorrelated in the respective energy window.

The fit results are shown in figure 9 and listed in table 8 . The ${ }^{210} \mathrm{Po}$ component is modeled with a combination of $\mathrm{p}^{+}$contact thicknesses from 400 to $600 \mathrm{~nm}$ for the M1-enrBEGe data set and from 300 to $700 \mathrm{~nm}$ for the M1-enrCoax data set in steps of $100 \mathrm{~nm}$. Further ${ }^{210} \mathrm{Po}$ components are rejected by a Bayes factor analysis. Impurities belonging to the ${ }^{226} \mathrm{Ra}$ chain are mostly located on ANG2 and thus a fit of the M1-enrCoax data set using a single $\mathrm{p}^{+}$thickness describes this component well. For the M1-enrBEGe data set we observe a very small number of counts for the ${ }^{226} \mathrm{Ra}$ chain, therefore, also in this case a single com-

\footnotetext{
${ }^{8}$ The calibration curves are accurate on the sub-keV level up to the highest $\gamma$ energy of about $2.6 \mathrm{MeV}$ emitted by the ${ }^{228} \mathrm{Th}$ calibration sources. Although no major non-linearity effects were found the same accuracy cannot be guaranteed at $6 \mathrm{MeV}$. Deviations from linearity at this energy are within $10 \mathrm{keV}$, hence, we increase the bin size in the higher energy range.
} 


\begin{tabular}{|c|c|c|c|c|}
\hline data set & component & $\begin{array}{c}\text { contact } \\
{[\mathrm{nm}]}\end{array}$ & $\begin{array}{c}\text { global mode } \\
\text { [cts] }\end{array}$ & $\begin{array}{l}\text { marg. mode } \\
68 \% \text { C.I. }[\mathrm{cts}]\end{array}$ \\
\hline \multirow{6}{*}{ M1-enrBEGe } & \multirow{4}{*}{${ }^{210} \mathrm{Po}$} & 400 & 49 & $50[34,76]$ \\
\hline & & 500 & 162 & $165[107,222]$ \\
\hline & & 600 & 346 & $342[278,391]$ \\
\hline & & comb. & - & $555[523,586]$ \\
\hline & \multirow{2}{*}{$\begin{array}{l}{ }^{226} \text { Ra chain } \\
\text { energy-degraded }\end{array}$} & 500 & 20 & $20[15,29]$ \\
\hline & & - & - & $845[698,948]$ \\
\hline \multirow{8}{*}{ M1-enrCoax } & \multirow{6}{*}{${ }^{210} \mathrm{Po}$} & 300 & 167 & $165[140,208]$ \\
\hline & & 400 & 363 & $368[272,430]$ \\
\hline & & 500 & 182 & $175[83,338]$ \\
\hline & & 600 & 433 & $420[233,582]$ \\
\hline & & 700 & 404 & $410[295,537]$ \\
\hline & & comb. & - & $1555[1511,1609]$ \\
\hline & ${ }^{226}$ Ra chain & 100 & 58 & $59[49,70]$ \\
\hline & energy-degraded & - & - & $485[426,599]$ \\
\hline
\end{tabular}

Table 8. Fit results of the $\alpha$ events background analysis for the M1-enrBEGe and M1-enrCoax data sets. Values are given in counts in the full PDF range from $40 \mathrm{keV}$ to $8000 \mathrm{keV}$.

ponent is sufficient. We determine a best-fit value of $100 \mathrm{~nm}$ and $500 \mathrm{~nm}$, respectively. The estimated $p$-value for M1-enrBEGe is 0.2 whereas the $p$-value for M1-enrCoax is 0.3 . The dominant spectral component below $4.5 \mathrm{MeV}$ is due to degraded $\alpha$ events which extends down to the ROI.

\section{B.1 Time distribution of $\alpha$ events}

The time distribution of ${ }^{210} \mathrm{Po}$ decays is well known to be exponential, however, in the presence of a ${ }^{210} \mathrm{~Pb}$ contamination a constant contribution can also be observed. ${ }^{210} \mathrm{~Pb}$, decaying to ${ }^{210} \mathrm{Po}$, feeds a constant ${ }^{210} \mathrm{Po}$ component once their decay rates stabilize in a secular equilibrium. To disentangle the two we fit the time distribution of events with energies between $3.5 \mathrm{MeV}$ and $5.25 \mathrm{MeV}$ with a constant $C$ and an exponential function:

$$
f(t)=C+N \exp \left(-\frac{\log 2}{T_{1 / 2}} t\right)
$$

where $T_{1 / 2}=(138.4 \pm 0.2)$ days is the half-life of ${ }^{210} \mathrm{Po}$. We use a Poisson likelihood function corrected for data acquisition dead time [50] and model the time bin content as follows

$$
\nu_{i}=f_{i}^{\mathrm{LT}}\left\{C \delta t+N \tau\left[\exp \left(-\frac{t_{0}+i \delta t}{\tau}\right)-\exp \left(-\frac{t_{0}+(i+1) \delta t}{\tau}\right)\right]\right\}
$$



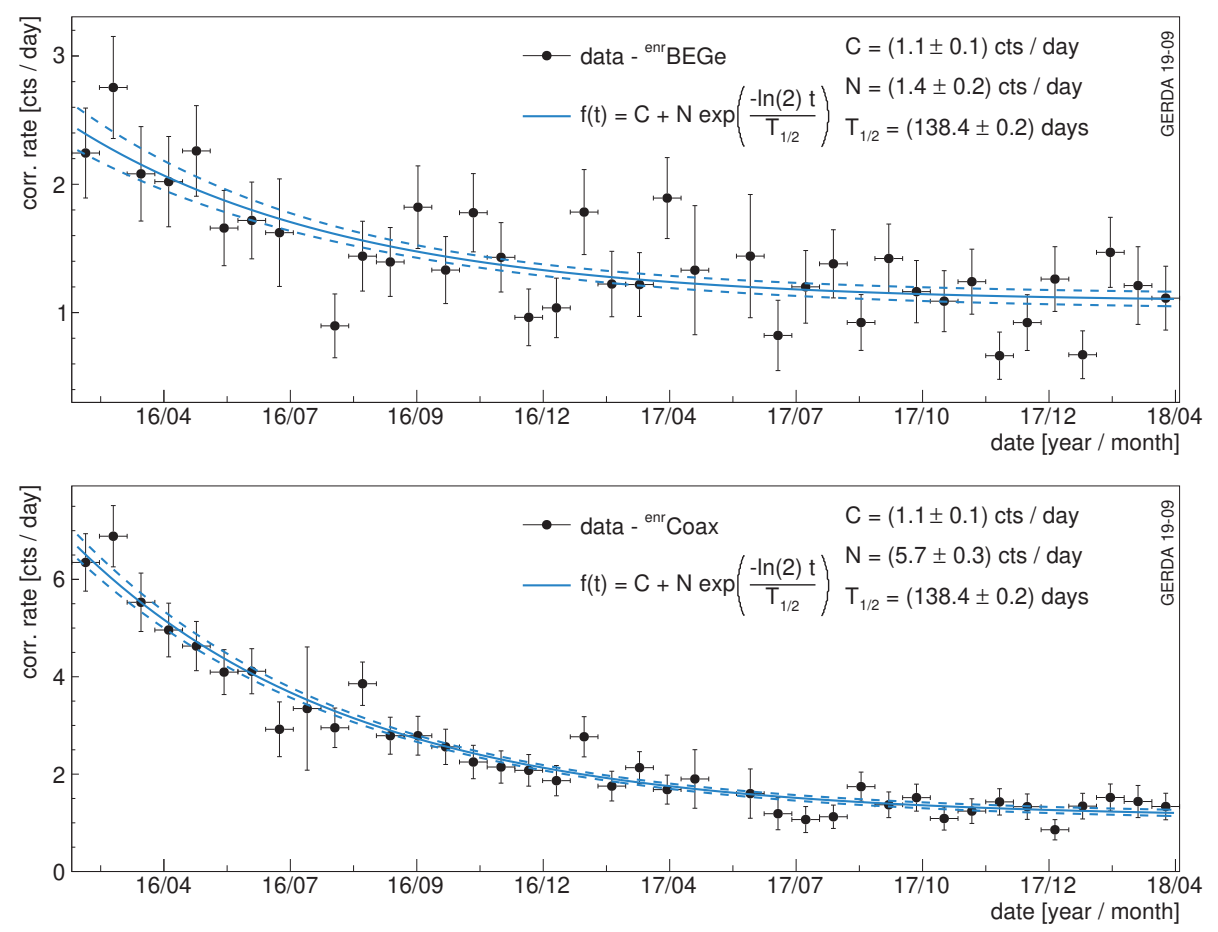

Figure 10. $\alpha$ events time distribution in $[3500,5250] \mathrm{keV}$ with a binning of 20 days for $27{ }^{\mathrm{enr}}$ BEGe (top) and $7{ }^{\text {enr }}$ Coax (bottom) detectors.

$C$ and $N$ are the amplitudes of the constant and the exponentially decaying components and are the only two free fit parameters. $f_{i}^{\mathrm{LT}}$ is the live-time fraction in time-bin $i$ which is estimated from injected test pulser events, $\delta t$ is the time-bin width and $\tau=T_{1 / 2} / \log 2$.

The log-likelihood can be written as a sum:

$$
\log \mathcal{L}_{\alpha}^{\mathrm{time}}(C, N \mid n)=\sum_{i=1}^{N_{\text {bins }}} n_{i} \cdot \log \nu_{i}-\nu_{i}-\log n_{i} !
$$

We select only detectors that were $\mathrm{ON}$ or in anti-coincidence mode ${ }^{9}$ in the full data taking period. In this way we avoid bias due to selection or deselection of particularly contaminated detectors. Furthermore, we exclude the initial data-taking period between December 2015 to January 2016 from the following analysis because of detector instabilities after the Phase II upgrade works. The analyzed data span from $25^{\text {th }}$ January 2016 to $3^{\text {rd }}$ April 2018 and are split into two data sets according to detector type, containing $27{ }^{\mathrm{enr}} \mathrm{BEGe}$ and 7

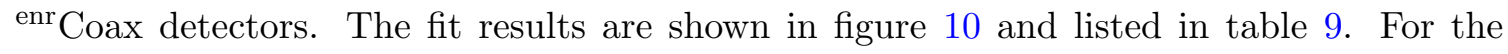

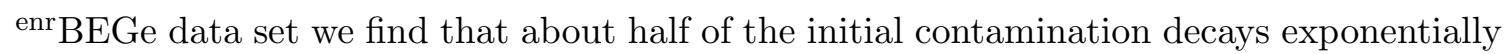

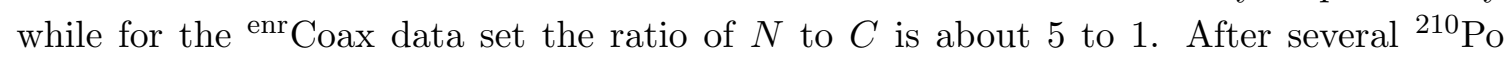
half-lives we expect a stable rate of $\sim 1 \alpha$ /day in either data set.

\footnotetext{
${ }^{9}$ Detectors in anti-coincidence are not well energy-calibrated and generally discarded in data analysis. Here, we are not interested in the precise energy of an event because the selected energy window is large with respect to a possible miscalibration.
} 


\begin{tabular}{|ccccc|}
\hline parameter & data & units & global mode & $\begin{array}{c}\text { marg. mode } \\
68 \% \text { C.I. }\end{array}$ \\
\hline \multirow{2}{*}{$C$} & ${ }^{\text {enr BEGe }}$ & cts/day & 1.06 & $1.05[1.00,1.12]$ \\
& ${ }^{\text {enr Coax }}$ & & 1.09 & $1.09[1.02,1.16]$ \\
\multirow{2}{*}{$N$} & ${ }^{\text {enr BEGe }}$ & & 1.32 & $1.33[1.13,1.53]$ \\
& ${ }^{\text {enr Coax }}$ & cts/day & 5.71 & $5.70[5.42,6.01]$ \\
\hline
\end{tabular}

Table 9. Results of the $\alpha$ events time distribution analysis in $[3500,5250] \mathrm{keV}$ with a binning of 20 days for $27{ }^{\mathrm{enr}} \mathrm{BEGe}$ and $7{ }^{\mathrm{enr}}$ Coax detectors.

\section{Monte Carlo simulations and probability density functions}

Background components that were identified in the energy spectra (see section 2) or in radio-purity screening measurements [5] are simulated using the MAGE software [18] based on GEANT4 [19-21].

The GERDA Phase II detectors, their arrangement in seven strings as well as the LAr instrumentation are implemented into MAGE. A graphic rendering of the relevant implemented hardware components is presented in figure 1 .

Simulations of radioactive contaminations in the following hardware components are performed: in the bulk and on the $\mathrm{p}^{+}$and $\mathrm{n}^{+}$surfaces of the germanium detectors, in the LAr, detector holder bars and plates, nylon mini-shrouds, LAr veto system (i.e. the fiber shroud, SiPMs, copper shrouds and photomultipliers) and in the signal and high-voltage flexible flat cables. The primary spectrum of the two electrons emitted in the $2 \nu \beta \beta$ decay is sampled according to the distribution given in reference [22] implemented in DECAY0 [23]. Note that the thickness of the detector assembly components are significantly smaller than the mean free path of the relevant simulated $\gamma$ particles in the given material, thus, no significant difference can be expected between the resulting spectra of bulk and surface contaminations. The detectors $\mathrm{n}^{+}$contact thicknesses are implemented according to the values reported in references $[13,14]$.

The ${ }^{42} \mathrm{~K}$ decays (except for surface contaminations) are simulated homogeneously distributed in the relevant LAr volume. The following LAr volumes are chosen for the background model: the first is a cylinder centered on the detector array $(h=250 \mathrm{~cm}$, $r=100 \mathrm{~cm}$, simply referred to as "homogeneous" or abbreviated to "hom." in the following) subsequently divided into the volume enclosed by the mini-shrouds and the remaining one (outside the mini-shrouds); the second is a cylinder $(h=100 \mathrm{~cm}, r=25 \mathrm{~cm})$ positioned just above the array and the remaining seven are smaller cylinders $(h=20 \mathrm{~cm}, r=5 \mathrm{~cm})$, each one positioned just above each of the seven detector strings.

On top of the MAGE simulations a post-processing step is performed to compute the Probability Density Functions (PDFs) used to model the GERDA data in the statistical analysis. This includes folding in run-time dependent information, i.e. the detector status in each physics run, the finite energy resolution and threshold of each detector. All PDFs presented in the following are computed using the run-time parameters of the data sets 


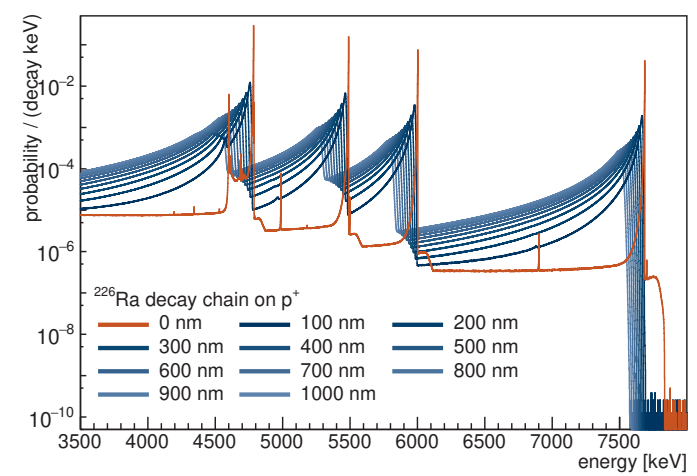

(a) $\alpha$ decays from the ${ }^{226} \mathrm{Ra}$ decay sub-chain $\left({ }^{226} \mathrm{Ra},{ }^{222} \mathrm{Rn},{ }^{218} \mathrm{Po}\right.$ and $\left.{ }^{214} \mathrm{Po}\right)$ on the detectors $\mathrm{p}^{+}$contact surface for different depths of the inactive contact layer. The isotope contamination is assumed to halve at each decay step, because of the recoil of the nuclei in LAr.

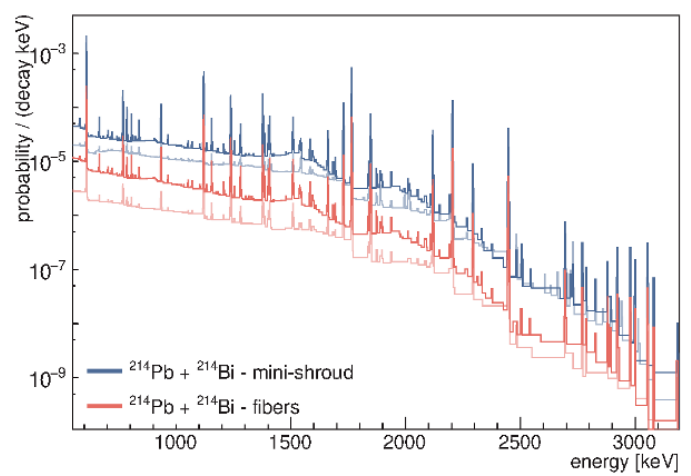

(b) ${ }^{214} \mathrm{~Pb}$ and ${ }^{214} \mathrm{Bi}\left({ }^{238} \mathrm{U}\right.$ chain) contaminations far from (fiber-shroud) and close to (minishrouds) the detector array. A variable binning is adopted for visualization purposes.

Figure 11. PDFs in the full energy domain. All PDFs are normalized to the number of simulated primary decays.

described in section 2. A selection of the PDFs projected in energy space and normalized to the number of simulated primary decays, are displayed in figure 3 and figure 11.

For the potassium tracking analysis PDFs binned in detector space are used to model the data. The rotationally symmetric single-detector PDFs for the ${ }^{40} \mathrm{~K}$ and ${ }^{42} \mathrm{~K}$ energy windows are shown in figure $3 \mathrm{f}$ and figure 12a. For two-detector events the same representation style as in figure 7 is used: projections of the two-dimensional histograms on their axis are summed, such that each two-detector event enters the final histogram twice, in the two bins associated to the respective detectors. They can be found in figure 12 together with the single-detector PDFs of the rotationally asymmetric components.

Common features can be noticed across the multitude of histogram shapes. The event rate in single-detector data is generally higher in coaxial detectors, due to their larger mass compared to BEGe detectors - maximal correlation between event rate and detector-bydetector exposure can be found in the $2 \nu \beta \beta \mathrm{PDF}$ in figure $3 \mathrm{f}$. This feature is generally lost in the two-detector data: the coaxial detectors larger volume allows to stop more efficiently $\gamma$ particles that would otherwise escape and eventually deposit energy in a second detector. Other similarities between different PDFs can be attributed to detectors live-times, like in the case of GD91C, which was inactive for a large fraction of the Phase II exposure and thus generally registers a low number of counts. The effects of asymmetrically distributed background contaminations are easily recognizable in the shape of the PDFs. Impurities located above the detector array are mostly seen by the upper most detectors in each string as can be seen for ${ }^{40} \mathrm{~K}$ in the front-end electronics in figure $12 \mathrm{a}$ and in figure $12 \mathrm{c}$ and for ${ }^{42} \mathrm{~K}$ above each mini-shroud (see figure $12 \mathrm{e}$ and figure $12 \mathrm{~d}$ ). Rotationally asymmetric components are mostly evident in a single string, see for example ${ }^{40} \mathrm{~K}$ in single mini-shrouds in figure $12 \mathrm{~b}$ and figure $12 \mathrm{~d}$. 


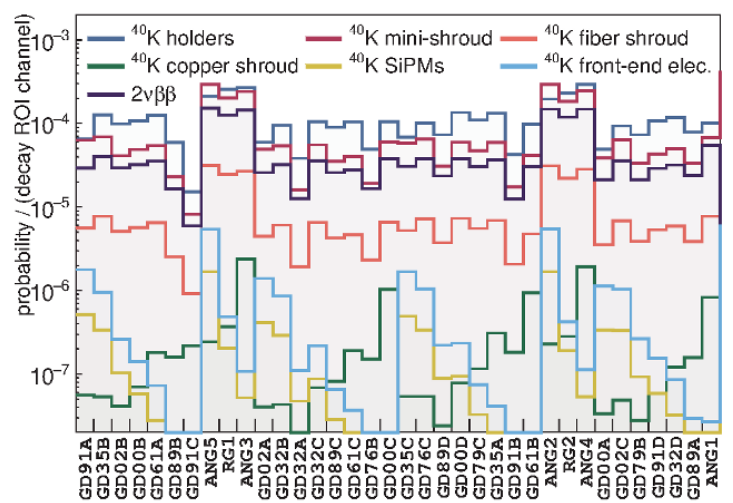

(a) ${ }^{40} \mathrm{~K}$ in different setup locations and $2 \nu \beta \beta$ in Ge, M1-K40 data set.

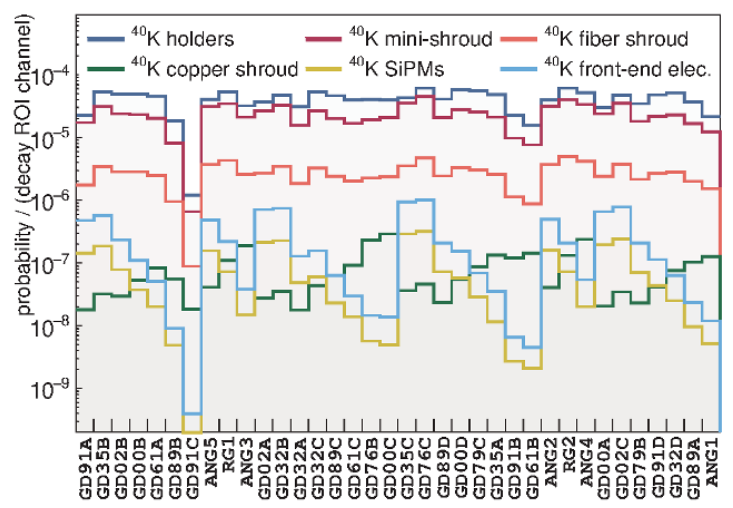

(c) ${ }^{40} \mathrm{~K}$ in different setup locations, M2-K40 data set.

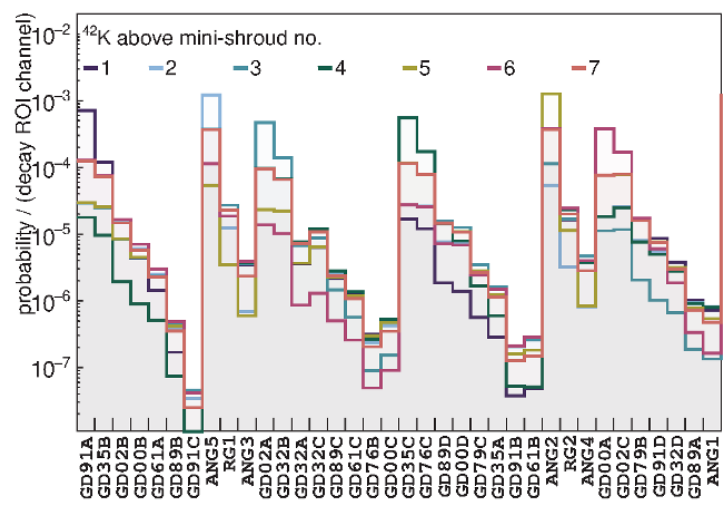

(e) ${ }^{42} \mathrm{~K}$ in LAr above each single mini-shroud, M1-K42 data set.

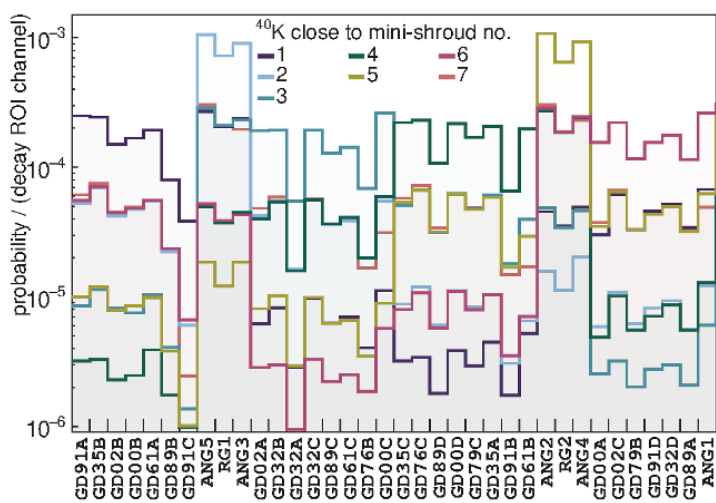

(b) ${ }^{40} \mathrm{~K}$ located close to each single mini-shroud, M1-K40 data set.

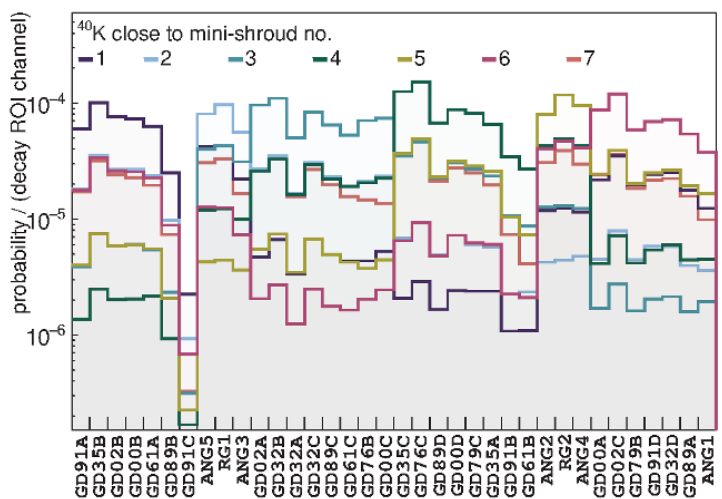

(d) ${ }^{40} \mathrm{~K}$ located close to each single mini-shroud, M2-K40 data set.

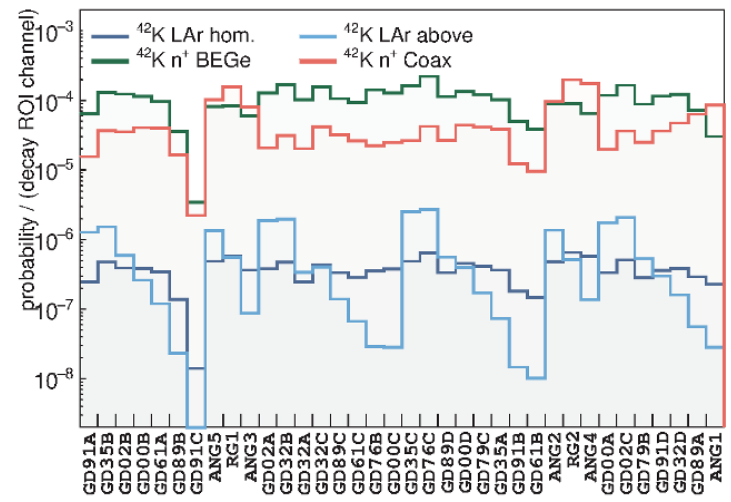

(f) ${ }^{42} \mathrm{~K}$ in different setup locations, M2-K42 data set.

Figure 12. PDFs binned in detector space for the potassium tracking analysis. All PDFs are normalized to the number of simulated primary decays. 
All $\alpha$ decays in the ${ }^{226} \mathrm{Ra}$ to ${ }^{210} \mathrm{~Pb}$ sub-chain and from ${ }^{210} \mathrm{Po}$ are simulated on the $\mathrm{p}^{+}$ detector surface separately and for different thicknesses of the $\mathrm{p}^{+}$electrode. The ${ }^{226} \mathrm{Ra}$ chain is simulated together under the assumption that in each $\alpha$ decay half of the contamination is lost due to the recoil of the nucleus into the LAr. The resulting PDFs are displayed in figure 3e and figure 11a. The spectra exhibit a peak like structure with a pronounced low-energy tail. The maximum is shifted with respect to the full emission energy due to the thickness of the $\mathrm{p}^{+}$contact. The low-energy tail is characteristic for $\alpha$ decays; the $\alpha$ particle is susceptible to the change in the contact thickness when penetrating the detector surface under an incident angle and loses part of its energy before reaching the active detector volume.

Open Access. This article is distributed under the terms of the Creative Commons Attribution License (CC-BY 4.0), which permits any use, distribution and reproduction in any medium, provided the original author(s) and source are credited.

\section{References}

[1] R.N. Mohapatra and A.Y. Smirnov, Neutrino Mass and New Physics, Ann. Rev. Nucl. Part. Sci. 56 (2006) 569 [hep-ph/0603118] [INSPIRE].

[2] R.N. Mohapatra et al., Theory of neutrinos: A White paper, Rept. Prog. Phys. 70 (2007) 1757 [hep-ph/0510213] [INSPIRE].

[3] H. Päs and W. Rodejohann, Neutrinoless double beta decay, New J. Phys. 17 (2015) 115010.

[4] Gerda collaboration, The Gerda experiment for the search of $0 \nu \beta \beta$ decay in ${ }^{76}$ Ge, Eur. Phys. J. C 73 (2013) 2330 [arXiv:1212.4067] [InSPIRE].

[5] Gerda collaboration, Upgrade for Phase II of the Gerda experiment, Eur. Phys. J. C 78 (2018) 388 [arXiv: 1711.01452] [INSPIRE].

[6] B.J. Mount, M. Redshaw and E.G. Myers, Double- $\beta$-decay $Q$ values of ${ }^{74}$ Se and ${ }^{76}$ Ge, Phys. Rev. C 81 (2010) 032501 [INSPIRE].

[7] GERDA collaboration, Background-free search for neutrinoless double- $\beta$ decay of ${ }^{76}$ Ge with GERDA, Nature $\mathbf{5 4 4}$ (2017) 47 [arXiv:1703.00570] [INSPIRE].

[8] Gerda collaboration, Improved Limit on Neutrinoless Double- $\beta$ Decay of ${ }^{76}$ Ge from Gerda Phase II, Phys. Rev. Lett. 120 (2018) 132503 [arXiv: 1803.11100] [INSPIRE].

[9] Gerda collaboration, Probing Majorana neutrinos with double- $\beta$ decay, Science 365 (2019) 1445 [arXiv: 1909.02726] [INSPIRE].

[10] Gerda collaboration, Pulse shape discrimination for Gerda Phase I data, Eur. Phys. J. C 73 (2013) 2583 [arXiv: 1307.2610] [INSPIRE].

[11] A. Lubashevskiy et al., Mitigation of ${ }^{42} \mathrm{Ar} /{ }^{42} \mathrm{~K}$ background for the GERDA Phase II experiment, Eur. Phys. J. C 78 (2018) 15 [arXiv:1708.00226] [INSPIRE].

[12] GERDA collaboration, Production, characterization and operation of ${ }^{76} \mathrm{Ge}$ enriched BEGe detectors in Gerda, Eur. Phys. J. C 75 (2015) 39 [arXiv:1410.0853] [INSPIRE].

[13] Gerda collaboration, Characterization of $30^{76}$ Ge enriched Broad Energy Ge detectors for Gerda Phase II, Eur. Phys. J. C 79 (2019) 978 [arXiv:1901.06590] [InSPIRE]. 
[14] Gerda collaboration, The background in the $0 \nu \beta \beta$ experiment Gerda, Eur. Phys. J. C 74 (2014) 2764 [arXiv: 1306.5084] [INSPIRE].

[15] L. Vanhoefer, Limitations of Rare Event HPGe Experiments due to Muon-Induced Neutron Background, Ph.D. Thesis, Technische Universität München (2018).

[16] Gerda collaboration, Improvement of the energy resolution via an optimized digital signal processing in Gerda Phase I, Eur. Phys. J. C 75 (2015) 255 [arXiv: 1502.04392] [InSPIRE].

[17] T. Wester, Characterization of coincidence data of the GERDA experiment to search for double beta decays to excited states, Ph.D. Thesis, Technische Universität Dresden (2019).

[18] M. Boswell et al., MaGe - a Geant4-Based Monte Carlo Application Framework for Low-Background Germanium Experiments, IEEE Trans. Nucl. Sci. 58 (2011) 1212.

[19] Geant4 collaboration, Geant4: A Simulation toolkit, Nucl. Instrum. Meth. A 506 (2003) 250 [INSPIRE].

[20] J. Allison et al., Geant4 developments and applications, IEEE Trans. Nucl. Sci. 53 (2006) 270.

[21] J. Allison et al., Recent developments in Geant4, Nucl. Instrum. Meth. A 835 (2016) 186 [INSPIRE].

[22] V. Tretyak and Y. Zdesenko, Tables of double beta decay data, Atom. Data Nucl. Data Tabl. 61 (1995) 43.

[23] O.A. Ponkratenko, V.I. Tretyak and Y.G. Zdesenko, The Event generator DECAY4 for simulation of double beta processes and decay of radioactive nuclei, Phys. Atom. Nucl. 63 (2000) 1282 [nucl-ex/0104018] [INSPIRE].

[24] M.L. di Vacri et al., ICP MS selection of radiopure materials for the GERDA experiment, AIP Conf. Proc. 1672 (2015) 150001 [InSPIRE].

[25] G. Meierhofer, P. Kudejova, L. Canella, P. Grabmayr, J. Jochum and J. Jolie, Thermal neutron capture cross-section of ${ }^{76}$ Ge, Eur. Phys. J. A 40 (2009) 61.

[26] G. Meierhofer, P. Grabmayr, J. Jochum, P. Kudejova, L. Canella and J. Jolie, Thermal neutron capture cross section of ${ }^{74}$ Ge, Phys. Rev. C 81 (2010) 027603 [INSPIRE].

[27] G. Meierhofer, P. Grabmayr, L. Canella, P. Kudejova, J. Jolie and N. Warr, Prompt $\gamma$ rays in ${ }^{77} \mathrm{Ge}$ and ${ }^{75} \mathrm{Ge}$ after thermal neutron capture, Eur. Phys. J. A 48 (2012) 20 [INSPIRE].

[28] H.V. Klapdor-Kleingrothaus et al., Latest results from the Heidelberg-Moscow double beta decay experiment, Eur. Phys. J. A 12 (2001) 147 [hep-ph/0103062] [INSPIRE].

[29] IGEX collaboration, The IGEX ${ }^{76}$ Ge neutrinoless double beta decay experiment: Prospects for next generation experiments, Phys. Rev. D 65 (2002) 092007 [hep-ex/0202026] [INSPIRE].

[30] GerdA collaboration, Limits on uranium and thorium bulk content in GerDA Phase I detectors, Astropart. Phys. 91 (2017) 15 [arXiv:1611.06884] [INSPIRE].

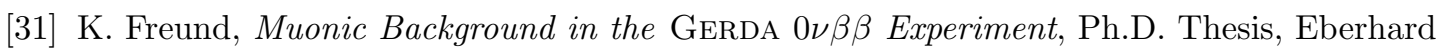
Karls Universität Tübingen (2014).

[32] C. Wiesinger, L. Pandola and S. Schönert, Virtual depth by active background suppression: Revisiting the cosmic muon induced background of GeRDA Phase II, Eur. Phys. J. C 78 (2018) 597 [arXiv : 1802.05040] [INSPIRE]. 
[33] I. Barabanov et al., Shielding of the GERDA experiment against external gamma background, Nucl. Instrum. Meth. A 606 (2009) 790 [inSPIRE].

[34] D.M. Mei, S.R. Elliott, A. Hime, V. Gehman and K. Kazkaz, Neutron inelastic scattering processes as a background for double-beta decay experiments, Phys. Rev. C 77 (2008) 054614 [arXiv:0704.0306] [INSPIRE].

[35] K. Winger, J. Feichter, M. Kalinowski, H. Sartorius and C. Schlosser, A new compilation of the atmospheric ${ }^{85}$ Krypton inventories from 1945 to 2000 and its evaluation in a global transport model, J. Environ. Radioactiv. 80 (2005) 183.

[36] A. Caldwell, D. Kollár and K. Kröninger, BAT: The Bayesian Analysis Toolkit, Comput. Phys. Commun. 180 (2009) 2197 [arXiv: 0808.2552] [INSPIRE].

[37] F. Beaujean, A. Caldwell, D. Greenwald, K. Kröninger and O. Schulz, BAT release, version 1.0.0, 10.5281/zenodo.1322675 (2018).

[38] F. Beaujean, A. Caldwell, D. Kollár and K. Kröninger, p-values for model evaluation, Phys. Rev. D 83 (2011) 012004 [arXiv:1011.1674].

[39] B. Lehnert, Search for $2 \nu \beta \beta$ Excited State Transitions and HPGe Characterization for Surface Events in GERDA Phase II, Ph.D. Thesis, Technische Universität Dresden (2016).

[40] R. Aggarwal and A. Caldwell, Error bars for distributions of numbers of events, Eur. Phys. J. Plus. 127 (2012) 24.

[41] Gerda collaboration, Results on Neutrinoless Double- $\beta$ Decay of ${ }^{76}$ Ge from Phase I of the Gerda Experiment, Phys. Rev. Lett. 111 (2013) 122503 [arXiv:1307.4720] [InSPIRE].

[42] Gerda collaboration, Measurement of the half-life of the two-neutrino double beta decay of ${ }^{76}$ Ge with the GERDA experiment, J. Phys. G 40 (2013) 035110 [arXiv:1212.3210] [INSPIRE].

[43] GERDA collaboration, Results on $\beta \beta$ decay with emission of two neutrinos or Majorons in ${ }^{76}$ Ge from GERda Phase I, Eur. Phys. J. C 75 (2015) 416 [arXiv:1501.02345] [InSPIRE].

[44] J.S. Díaz, Limits on Lorentz and CPT violation from double beta decay, Phys. Rev. D 89 (2014) 036002 [arXiv:1311.0930] [INSPIRE].

[45] LEGEND collaboration, The Large Enriched Germanium Experiment for Neutrinoless Double Beta Decay (LEGEND), AIP Conf. Proc. 1894 (2017) 020027 [arXiv:1709.01980] [INSPIRE].

[46] V. D'Andrea, Improvement of Performance and Background Studies in GERDA Phase II, Ph.D. Thesis, Gran Sasso Science Institute (GSSI) (2017).

[47] M.Z.M.J. Berger, J.S. Coursey and J. Chang, Stopping-Power $\&$ Range Tables for Electrons, Protons, and Helium Ions, NIST Standard Reference Database 124 (2017) .

[48] M.-M. Bé et al., Table of Radionuclides, vol. 4 of Monographie BIPM-5, Bureau International des Poids et Mesures, Pavillon de Breteuil, F-92310 Sèvres, France (2008).

[49] M. Agostini, Signal and background studies for the search of neutrinoless double beta decay in Gerda, Ph.D. Thesis, TechnischeV Universität München (2013).

[50] B.T. Cleveland, The analysis of radioactive decay with a small number of counts by the method of maximum likelihood, Nucl. Instrum. Meth. 214 (1983) 451 [INSPIRE]. 